Equações diferenciais funcionais do ponto de vista das equações de renovação

Vinícius de Castro Nunes de Siqueira 
SERVIÇO DE PÓS-GRADUAÇÃO DO ICMC-USP

Data de Depósito: 18 de Março de 2009

Assinatura:

\title{
Equações diferenciais funcionais do ponto de vista das equações de renovação
}

\author{
Vinícius de Castro Nunes de Siqueira
}

Orientador: Prof. Dr. Miguel Vinícius Santini Frasson

Dissertação apresentada ao Instituto de Ciências Matemáticas e de Computação - ICMC-USP, como parte dos requisitos para obtenção do título de Mestre em Ciências - Matemática.

USP - São Carlos

Março/2009 

- Hos mens pais.

cone anovi... 


\section{Agradecimentos}

Ao professor Miguel, por todo o conhecimento transmitido, dedicação, paciência, seriedade na orientação e, principalmente pelo apoio. Graças a você, tive forças para conseguir concluir este trabalho.

Aos meus pais, Cláudio e Aurora, pela oportunidade do estudo e pelo apoio e incentivo durante todas as etapas dos meus estudos, preocupando-se para que a minha permanência em São Carlos fosse a melhor possível. E a toda minha família, pela preocupação e incentivo.

A Isabela, pelo amor, apoio e compreensão no decorrer deste período.

A todos os meus amigos, pela amizade, auxílio, discussões e por todos os momentos de lazer e divertimento que passamos juntos.

A todos os professores do ICMC, pelo conhecimento, amizade e auxílio indispensáveis à minha formação. Em especial à professora Sandra pela orientação durante as minhas iniciações científicas, despertando em mim, o prazer pela pesquisa matemática.

A todos os funcionários do ICMC, pela atenção e por me proporcionarem um ambiente seguro e adequado ao estudo.

A CAPES, pelo apoio financeiro.

Enfim, a todos que colaboraram de forma direta ou indireta com este trabalho. 


\section{Resumo}

Estudamos a representação das equações diferenciais funcionais (EDF) lineares autônomas do tipo neutro como uma classe de equações de renovação, isto é, equações do tipo convolução. Utilizando ferramentas como a transformada de Laplace-Stieltjes, estudamos o comportamento assintótico das soluções destas equações quando $t \rightarrow \infty$. 


\section{Abstract}

We studied the representation of linear autonomous functional differential equations (FDE) as a class of renewall equations, that is, convolution-type equations. Using tools like the Laplace-Stieltjes trnsform, we obtained the asymptotic behaviour of those solutions as $t \rightarrow \infty$. 


\section{Sumário}

$\begin{array}{ll}\text { Introdução } & 1\end{array}$

1 Conceitos básicos 3

1.1 Notação e definições . . . . . . . . . . . . . . . . . 3

1.2 Convolução de funções e medidas de Borel . . . . . . . . . . 4

1.3 A transformada de Laplace-Stieltjes . . . . . . . . . . . . 7

2 Equações diferenciais funcionais e equações de renovação 13

2.1 Problema de valor inicial para EDF . . . . . . . . . . . . . 13

2.2 Equações de renovação . . . . . . . . . . . . . . . . . . . . . 14

2.2.1 A solução fundamental . . . . . . . . . . . . . . . 18

2.2 .2 Núcleo resolvente . . . . . . . . . . . . . . . . . . . 19

2.3 Representação de uma EDF como uma equação de renovação 22

2.4 O espaço das funções forçantes . . . . . . . . . . . . . . 28

2.5 Resolução de EDF por transformadas de Laplace . . . . . . 29

3 Comportamento assintótico $\quad 33$

3.1 Estimativas para $\Delta(z)$ e quantidades relacionadas . . . . . . 33

3.2 Comportamento assintótico para $t \rightarrow \infty$. . . . . . . . 45

$\begin{array}{ll}\text { Bibliografia } & \mathbf{5 3}\end{array}$ 


\section{Introdução}

Nesta dissertação, estabelecemos uma conexão entre equações diferenciais funcionais e uma classe de equações de renovação. As principais referências utilizadas neste trabalho são a tese de Frasson [4] e o livro de Hale \& Verduyn Lunel [5].

A idéia básica é representar um problema de valor inicial (PVI) de uma equação diferencial funcional como uma equação de renovação, estudar o espaço das funções forçantes, encontrar a solução da equação de renovação utilizando as tranformadas de Laplace-Stieltjes e então, estudar o comportamento assintótico de tais soluções. O foco principal do trabalho está em equações do tipo neutro, uma vez que encontramos trabalhos similares para equações retardadas em Diekmann et al. [3].

O trabalho está organizado da seguinte maneira:

No Capítulo 1 elaboramos os conceitos básicos, notações e definições da teoria, os quais serão utilizados no restante do texto.

No Capítulo 2 estudamos o problema de representação de uma EDF como equação de renovação, analisamos o espaço das funções forçantes e um método de resolução da equação de renovação utilizando transformadas de Laplace-Stieltjes.

Finalmente, no Capítulo 3 estudamos algumas estimativas relacionadas com as soluções da equação de renovação e o seu comportamento assintótico. 


\section{Capítulo 1}

\section{Conceitos básicos}

\subsection{Notação e definições}

Neste trabalho, denotaremos por $\mathbb{C}^{n}$ o conjunto dos vetores coluna com $n$ entradas complexas. Assim, todo vetor linha $\gamma \operatorname{com} n$ entradas em $\mathbb{C}$ pode ser identificado com um funcional em $\mathbb{C}^{n}$ dado por $v \mapsto \gamma v$. Assim, o conjunto dos vetores linha será denotado por $\mathbb{C}^{n *}$. O espaço das matrizes $n \times n$ será denotado por $\mathbb{C}^{n \times n}$. Em qualquer espaço métrico $M$ com métrica $d$, denotamos a bola aberta com centro $x$ e raio $r,\{y \in M$ tais que $d(x, y)<r\}$ por $B(x, r)$ e a bola fechada com centro $x$ e raio $r,\{y \in M$ tais que $d(x, y) \leqslant$ $r\}$ por $\bar{B}(x, r)$.

Considere $\mathcal{C} \stackrel{\text { def }}{=} \mathcal{C}\left([-r, 0], \mathbb{C}^{n}\right)$ o espaço de Banach das funções contínuas de $[-r, 0](r>0)$ com valores em $\mathbb{C}^{n}$ com a norma uniforme, ou seja, se $x \in$ $\mathcal{C}\left([-r, 0], \mathbb{C}^{n}\right)$ então $\|x\|=\sup _{-r \leqslant t \leqslant 0}|x(t)|$. De uma aplicação do teorema de representação de Riesz (veja, por exemplo Rudin [8] ou Royden [7]), podemos representar toda aplicação linear limitada $L: \mathcal{C} \rightarrow \mathbb{C}^{n}$ por

$$
L \varphi=\int_{0}^{r} d \eta(\theta) \varphi(-\theta)
$$

onde $\eta$ é uma função de variação limitada em $[0, r]$ normalizada tal que $\eta(0)=0$ e $\eta$ é contínua pela direita em $(0, r)$ com valores no espaço de matrizes $\mathbb{C}^{n \times n}$. Denotamos este conjunto de funções por $N B V\left([0, r], \mathbb{C}^{n \times n}\right)$. Assim definida, $\eta$ pode ser estendida em $\mathbb{R}$ por $\eta(\theta)=0$ se $\theta<0$ e $\eta(\theta)=\eta(r)$ se $\theta>r$. Funções $\alpha: \mathbb{R}_{+} \rightarrow \mathbb{C}^{n \times n}$ tais que a restrição de $\alpha$ a $[0, R]$ pertence 
a $N B V\left([0, R], \mathbb{C}^{n \times n}\right)$, para todo $R>0$, são chamadas funções localmente $N B V$ e o conjunto delas é denotado $N B V_{\text {loc }}\left(\mathbb{R}_{+}, \mathbb{C}^{n \times n}\right)$.

O teorema de representação de Riesz estabelece que todo funcional linear limitado $f: \mathcal{C} \rightarrow \mathbb{C}$ pode ser escrito na forma

$$
f(\varphi)=\int_{0}^{r} d \psi(\theta) \varphi(-\theta) \stackrel{\text { def }}{=}\langle\psi, \varphi\rangle
$$

onde $\psi \in N B V\left([0, r], \mathbb{C}^{n *}\right)$. Portanto, podemos representar o espaço dual de $\mathcal{C}$ por $\mathcal{C}^{*}=N B V\left([0, r], \mathbb{C}^{n *}\right)$.

Usaremos a notação comum na teoria de equações retardadas, ou seja, para uma função $x$ de $[-r, \infty)$ para algum espaço de Banach $X$, definimos $x_{t}:[-r, 0] \rightarrow X$ por $x_{t}(\theta)=x(t+\theta)$, com $-r \leqslant \theta \leqslant 0$ e $t \geqslant 0$.

A notação utilizada para a derivada de uma função $f$ será $\dot{f}$. A matriz adjunta de $A$ será denotada por adj $A$. Além disso, a linha $\{z \in \mathbb{C}: \operatorname{Re} z=$ $\gamma\}$, será denotada por $(\gamma)$ e definiremos $\int_{(\gamma)} \ldots d z$ como o valor principal da integral $\lim _{\omega \rightarrow \infty} \int_{\gamma-i \omega}^{\gamma+i \omega} \ldots d z$. A função de Heaviside $h_{y}$ é definida por $h_{y}(\theta)=0$ se $\theta<y$ e $h_{y}(\theta)=1$ se $\theta \geqslant y$.

No restante deste capítulo, veremos alguns resultados necessários para desenvolver a teoria das equações de renovação. Esses resultados foram retirados do livro de Salamon [9] e Widder [11] com pequenas modificações.

\subsection{Convolução de funções e medidas de Borel}

O primeiro resultado é uma coletânea de resultados menores sobre convoluções entre funções e medidas de Borel. Estes resultados encontram-se em $[9]$.

Observação 1.1 .

1. Os elementos de $L^{p}\left([0, R], \mathbb{C}^{n}\right)$ podem ser representados por classes de equivalência de funções $x$ em $[0, R]$ tais que a integral de Lebesgue de $|x|^{p}$ sobre $[0, R]$ existe e é finita, onde $x$ é equivalente a $y$ se a integral de Lebesgue de $|x-y|^{p}$ sobre $[0, R]$ é nula. Para $R>r$, podemos mergulhar $N B V\left([0, r], \mathbb{C}^{n \times n}\right)$ em $N B V\left([0, R), \mathbb{C}^{n \times n}\right)$ extendendo $\psi \in$ $N B V\left([0, r], \mathbb{C}^{n \times n}\right)$ a $[0, R)$, definindo $\psi(t)=\psi(r)$ para $t \geqslant r$. Os 
espaços de funções $N B V\left([0, R), \mathbb{C}^{n}\right)$ e $\mathcal{C}\left([0, R], \mathbb{C}^{n}\right)$ "são" subespaços vetoriais de $L^{p}\left([0, R), \mathbb{C}^{n}\right)$ para $1 \leqslant p \leqslant \infty$.

2. Tome $1 \leqslant p \leqslant \infty$ e $q$ tais que $\frac{1}{p}+\frac{1}{q}=1$ ( $\operatorname{se} p=\infty$, então $\left.q=1\right), f \in$ $L^{p}([0, R], \mathbb{C})$ e $g \in L^{q}([0, R], \mathbb{C})$. Deste modo, a convolução entre $f e$ $g$, definida por

$$
g * f(t)=\int_{0}^{t} g(s) f(t-s) d s=\int_{0}^{t} g(t-s) f(s) \quad 0 \leqslant t \leqslant R
$$

é contínua. Além disso, temos que $g * f(t)=f * g(t)$.

3. Todo $\alpha \in N B V\left([0, R), \mathbb{C}^{n \times n}\right)$ representa uma medida de Borel em $\mathbb{C}^{n}$ sem massa fora de $[0, R)$. Esta medida será denotada por $d \alpha$. Para qualquer função $f:[0, R] \rightarrow \mathbb{C}^{n}, d \alpha$-integrável, denotamos a integral de $f$ com respeito à medida $d \alpha$ por

$$
d \alpha(f)=\int_{0}^{R} d \alpha(t) f(t)
$$

4. Seja $\alpha \in N B V\left([0, R), \mathbb{C}^{n \times n}\right)$ e $f \in L^{p}\left([0, R], \mathbb{C}^{n}\right)$. Então, definimos a convolução entre a medida da e a função $f$,

$$
d \alpha * f(t)=\int_{0}^{t} d \alpha(s) f(t-s) d s
$$

para quase todo $t \in[0, R]$. Temos que $d \alpha * f \in L^{p}\left([0, R], \mathbb{C}^{n}\right)$. Se $f \in L^{p}\left([0, R], \mathbb{C}^{n *}\right)$, definimos $f * d \alpha$ por

$$
\int_{0}^{t} f(t-s) d \alpha(s)
$$

uma vez que $f$ é um vetor linha. Com isto, temos a seguinte propriedade:

$$
(d \alpha * f)^{T}=f^{T} * d \alpha^{T}
$$

5. A aplicação $f \mapsto d \alpha * f$ mapeia $\mathcal{C}_{R} \stackrel{\text { def }}{=}\left\{g \in \mathcal{C}\left([0, R], \mathbb{C}^{n}\right): g(0)=0\right\}$ em si mesmo e mapeia $N B V\left([0, R), \mathbb{C}^{n}\right)$ em si mesmo. No entanto, $\mathcal{C}\left([0, R], \mathbb{C}^{n}\right)$ é aplicado em si mesmo por $f \mapsto d \alpha * f$ se e somente se $\alpha$ é contínua. 
Mostremos que $f \mapsto d \alpha * f$ aplica $\mathcal{C}_{R}$ em $\mathcal{C}_{R}$. Se $f$ é contínua em um intervalo $[a, b]$ e $f$ é extendida para $t \in \mathbb{R}$ por $f(t)=f(a), t<a$ e $f(t)=f(b), t>b$, então $|f(t)-f(t+\delta)| \rightarrow 0$ uniformemente, quando $\delta \rightarrow 0$. Seja $f$ contínua em $[0, R] \operatorname{com} f(0)=0$ e defina $h(t)=d \alpha * f(t)$. Para $\delta>0$,

$$
\begin{aligned}
h(t+\delta)-h(t)= & \int_{0}^{t+\delta} d \alpha(s) f(t+\delta-s)-\int_{0}^{t} d \alpha(s) f(t-s) \\
= & \underbrace{\int_{0}^{t} d \alpha(s)(f(t+\delta-s)-f(t-s))}_{I_{1}(\delta)} \\
& +\underbrace{\int_{t}^{t+\delta} d \alpha(s) f(t+\delta-s)}_{I_{2}(\delta)} .
\end{aligned}
$$

Portanto, $h(t+\delta)-h(t)=I_{1}(\delta)+I_{2}(\delta)$. Quando $\delta \rightarrow 0^{+}$, temos que $f(t+\delta-s)-f(t-s) \rightarrow 0$ e então $I_{1}(\delta) \rightarrow 0$. Também podemos ver que, em $I_{2}(\delta), f$ é integrada sobre $(0, \delta]$. Portanto, uma vez que $f(0)=0$, temos que $I_{2}(\delta) \rightarrow 0$. Então, $h(t+\delta)-h(t) \rightarrow 0$ quando $\delta \rightarrow 0^{+}$, de onde segue que $h$ é contínua. Também $h(0)=0$. Logo $f \mapsto d \alpha * f$ aplica $\mathcal{C}_{R}$ em $\mathcal{C}_{R}$.

Para $f \in \mathcal{C}\left([0, R], \mathbb{C}^{n}\right)$ tal que $f(0) \neq 0$, definimos $f_{0} \in \mathcal{C}_{R}$ por $f_{0}(\cdot) \stackrel{\text { def }}{=}$ $f(\cdot)-f(0)$. Da primeira parte temos que $d \alpha * f_{0} \in \mathcal{C}_{R}$. Mas

$$
d \alpha * f=d \alpha *\left[f_{0}+f(0)\right]=d \alpha * f_{0}+d \alpha * f(0)=d \alpha * f_{0}+\alpha \cdot f(0) .
$$

Segue que $d \alpha * f \in \mathcal{C}$ se e somente se último membro de (1.3) for contínuo, que ocorre se e somente se $\alpha$ for uma função contínua.

6. Se $f$ é contínua, então (1.2) pode ser vista como uma integral de Stieltjes. Pelo Teorema de Representação de Riesz e usando o item $5, N B V\left([0, R), \mathbb{C}^{n \times n}\right)$ é (isometricamente isomorfo a) o espaço dual de $\mathcal{C}_{R}$

7. Para $\alpha, \beta \in N B V\left([0, R), \mathbb{C}^{n \times n}\right)$, a convolução $d \alpha * d \beta$ das medidas de Borel $d \alpha$ e $d \beta$ restritas ao intervalo $[0, R)$ é dada pela relação

$$
[d \alpha * d \beta](g)=\int_{0}^{R} \int_{0}^{R-s} d \beta(t) d \alpha(s) g(t+s)
$$




$$
=\int_{0}^{R} \int_{0}^{R-t} d \alpha(s) d \beta(t) g(t+s)
$$

para $g \in L^{p}\left([0, R], \mathbb{C}^{n}\right)$. Além disso, se $\alpha, \beta \in N B V\left([0, R), \mathbb{C}^{n \times n}\right)$, então $d \alpha * \beta \in N B V\left([0, R), \mathbb{C}^{n \times n}\right), d \alpha * \beta=\alpha * d \beta$ e

$$
d[\alpha * d \beta]=d \alpha * d \beta
$$

8. Se $f \in L^{p}\left([0, R], \mathbb{C}^{n}\right)$, vale a seguinte desigualdade

$$
\|d \alpha * f\|_{p} \leqslant \operatorname{Var}_{[0, R)} \alpha \cdot\|f\|_{p}
$$

\subsection{A transformada de Laplace-Stieltjes}

Utilizando as técnicas das transformadas de Laplace, o estudo das equações de renovação fica mais fácil, uma vez que transformamos convoluções de funções e medidas em produtos de funções complexas.

Definição 1.2 (Funções e medidas $\gamma$-exponencialmente limitadas). Uma função $g$ localmente integrável com domínio de definição $\mathbb{R}_{+}$é dita ser $\gamma$ exponencialmente limitada se existe $C>0$ tal que

$$
|g(t)| \leqslant C e^{\gamma t}
$$

Uma medida $d \alpha$ em $\mathbb{R}_{+}$, representada por uma função $\alpha \in N B V_{\text {loc }}$ é dita ser $\gamma$-exponencialmente limitada se sua função variação total é $\gamma$-exponencialmente limitada.

Definição 1.3 (Transformada de Laplace). Considere $g$ uma função localmente integrável com domínio de definição $\mathbb{R}_{+}$e $\gamma$-exponencialmente limitada. A transformada de Laplace de $g$, denotada $\mathcal{L}(g)(z)$, é definida por

$$
\mathcal{L}(g)(z) \stackrel{\text { def }}{=} \int_{0}^{\infty} e^{-z t} g(t) d t
$$

para todo $z \in \mathbb{C}$ tal que $\operatorname{Re} z>\gamma$.

Definição 1.4 (Transformada de Laplace-Stieltjes). Seja $d \alpha$ uma medida $\gamma$ exponencialmente limitada. Definimos a transformada de Laplace-Stieltjes de $d \alpha$ por

$$
\mathcal{L}(d \alpha)(z) \stackrel{\text { def }}{=} \int_{0}^{\infty} e^{-z t} d \alpha(t) .
$$

para todo $z \in \mathbb{C}$ tal que $\operatorname{Re} z>\gamma$. 
Em particular, se $\alpha$ é de variação limitada em $[0, r]$, segue que $d \alpha$ é $\gamma$ exponencialmente limitada para todo $\gamma \in \mathbb{R}$. Desta forma $\mathcal{L}(d \alpha)(z)$ é uma função inteira, uma vez que o intervalo de integração se reduz a $[0, r]$.

Mostremos que se $\alpha$ e $\beta$ são funções $N B V$ e $\mathcal{L}(d \alpha)(z)=\mathcal{L}(d \beta)(z)$, então $\alpha=\beta$ e se $f$ e $g$ são funções $\gamma$-exponencialmente limitadas, com domínio $\mathbb{R}_{+}$, com $\mathcal{L}(f)(z)=\mathcal{L}(g)(z)$, para $z$ em um semiplano $(\operatorname{Re} z>\gamma)$, então $f(t)=g(t)$ para quase todo $t \in \mathbb{R}_{+}$.

De fato, se $\alpha$ e $\beta$ são funções $N B V$ tais que

$$
\mathcal{L}(d \alpha)(z)=\int_{0}^{\infty} e^{-z t} d \alpha(t)=\int_{0}^{\infty} e^{-z t} d \beta(t)=\mathcal{L}(d \beta)(z),
$$

então, para $z$, tal que $\operatorname{Re} z>\gamma$, temos

$$
\begin{aligned}
0 & =\int_{0}^{\infty} e^{-z t} d \alpha(t)-\int_{0}^{\infty} e^{-z t} d \beta(t) \\
& =\int_{0}^{\infty} e^{-z t}(d \alpha(t)-d \beta(t)) \\
& =\int_{0}^{\infty} e^{-z t} d(\alpha(t)-\beta(t)) .
\end{aligned}
$$

Portanto, $\int_{0}^{\infty} e^{-z t} d(\alpha(t)-\beta(t))=0$, de onde segue (veja, por exemplo o Teorema II.6.3 de Widder [11]) que $\alpha(t)=\beta(t)$. Agora, se

$$
\mathcal{L}(f)(z)=\int_{0}^{\infty} e^{-z t} f(t) d t=\int_{0}^{\infty} e^{-z t} g(t) d t=\mathcal{L}(g)(z)
$$

então, tomando $\alpha(t)=\int_{0}^{t} f(u) d u$ e $\beta(t)=\int_{0}^{t} g(u) d u$ temos que vale (1.9). Logo $\alpha(t)=\beta(t)$ o que implica que $f(t)=g(t)$ para quase todo $t \in \mathbb{R}_{+}$, obtendo assim o resultado.

Lema 1.5. Sejam $f$ e $g$ funções $\gamma$-exponencialmente limitadas a valores em C. Para $z$ tal que $\operatorname{Re} z \geqslant \gamma$ temos que

$$
\mathcal{L}(f * g)(z)=\mathcal{L}(f)(z) \mathcal{L}(g)(z)
$$

Se $f$ assume valores em $\mathbb{C}^{n}$ e $\alpha \in N B V\left([0, \infty), \mathbb{C}^{n \times n}\right)$ é $\gamma$-exponencialmente limitada, temos que

$$
\mathcal{L}(d \alpha * f)(z)=\mathcal{L}(d \alpha)(z) \mathcal{L}(f)(z)
$$


Demonstração. De fato,

$\mathcal{L}(f * g)(z)=\int_{0}^{\infty} e^{-z t} \int_{0}^{\infty} f(s) g(t-s) d s d t=\int_{0}^{\infty} \int_{0}^{\infty} e^{-z t} f(s) g(t-s) d s d t$ e, considerando a mudança de variáveis $(t, s) \mapsto(u, v)$, tal que $s=v, t=$ $u+v$, temos

$$
\begin{aligned}
\mathcal{L}(f * g)(z) & =\int_{0}^{\infty} \int_{0}^{\infty} e^{-z(u+v)} f(v) g(u) d v d u \\
& =\int_{0}^{\infty} e^{-z v} f(v) d v \int_{0}^{\infty} e^{-z u} g(u) d u=\mathcal{L}(f)(z) \mathcal{L}(g)(z)
\end{aligned}
$$

Analogamente, mostramos que $\mathcal{L}(d \alpha * f)(z)=\mathcal{L}(d \alpha)(z) \mathcal{L}(f)(z)$, obtendo assim o resultado.

Além disso, para um função $f$ com valores em $\mathbb{C}^{n}$, definida e de variação limitada em $[0, r]$ e constante em $[r, \infty)$, temos a seguinte identidade

$$
\mathcal{L}(f)(z)=\frac{1}{z}(f(0)+\mathcal{L}(d f)(z)) .
$$

De fato,

$$
\begin{aligned}
\mathcal{L}(f)(z)=\int_{0}^{\infty} e^{-z t} f(t) d t & =\lim _{A \rightarrow \infty}\left[-\left.\frac{1}{z} e^{-z t} f(t)\right|_{0} ^{A}+\int_{0}^{A} \frac{1}{z} e^{-z t} d f(t)\right] \\
& =\frac{1}{z}\left(f(0)+\int_{0}^{r} e^{-z t} d f(t)\right)=\frac{1}{z}(f(0)+\mathcal{L}(d f)(z)) .
\end{aligned}
$$

Lema 1.6. Seja g uma função $\gamma$-exponencialmente limitada e localmente integrável. Então, para $\nu>\gamma$ e para $t>0$ temos a fórmula de inversão

$$
\frac{1}{2}(g(t+)+g(t-))=\lim _{\omega \rightarrow \infty} \frac{1}{2 \pi i} \int_{\nu-i \omega}^{\nu+i \omega} e^{z t} \mathcal{L}(g)(z) d z
$$

que, para $t=0$, torna-se

$$
\frac{1}{2} g(0+)=\lim _{\omega \rightarrow \infty} \frac{1}{2 \pi i} \int_{\nu-i \omega}^{\nu+i \omega} \mathcal{L}(g)(z) d z
$$

Demonstração. De fato, para qualquer valor de $t$, temos

$$
\begin{aligned}
\frac{1}{2 \pi i} \int_{\nu-i \omega}^{\nu+i \omega} e^{z t} \mathcal{L}(g)(z) d z & =\frac{1}{2 \pi i} \int_{\nu-i \omega}^{\nu+i \omega} e^{z t} d z \int_{0}^{\infty} e^{-z u} g(u) d u \\
& =\frac{1}{2 \pi i} \int_{\nu-i \omega}^{\nu+i \omega} \int_{0}^{\infty} e^{z(t-u)} g(u) d u d z
\end{aligned}
$$




$$
\begin{aligned}
& =\left.\frac{1}{2 \pi i} \int_{0}^{\infty} \frac{e^{z(t-u)}}{t-u} g(u)\right|_{\nu-i \omega} ^{\nu+i \omega} d u \\
& =\frac{1}{\pi} \int_{0}^{\infty} \frac{e^{\nu(t-u)} \operatorname{sen}(\omega(t-u))}{t-u} g(u) d u
\end{aligned}
$$

Agora, se $\varphi(u)$ é Lebesgue integrável em $(-\infty, \infty)$ e de variação limitada em alguma vizinhança de um ponto $t$, pelo Teorema II.7.2 de Widder [11], temos

$$
\lim _{T \rightarrow \infty} \frac{1}{\pi} \int_{-\infty}^{\infty} \frac{\varphi(u) \operatorname{sen}(T(t-u))}{t-u} d u=\frac{\varphi(t+)+\varphi(t-)}{2}
$$

Assim, tomando $g(u)=0$ em $(-\infty, 0)$, a função $g(u) e^{\nu(t-u)}$ é Lebesgue integrável em $(-\infty, \infty)$, logo

$$
\begin{aligned}
\lim _{\omega \rightarrow \infty} & \frac{1}{2 \pi i} \int_{\nu-i \omega}^{\nu+i \omega} e^{z t} \mathcal{L}(g)(z) d z \\
& =\lim _{\omega \rightarrow \infty} \frac{1}{\pi} \int_{0}^{\infty} \frac{e^{\nu(t-u)} \operatorname{sen}(\omega(t-u))}{t-u} g(u) d u=\frac{g(t+)+g(t-)}{2}
\end{aligned}
$$

Em $t=0, g(0-)=0, \log \mathrm{O}$

$$
\lim _{\omega \rightarrow \infty} \frac{1}{2 \pi i} \int_{\nu-i \omega}^{\nu+i \omega} \mathcal{L}(g)(z) d z=\frac{1}{2} g(0+) .
$$

A seguir, damos alguns resultados que garantem que a convolução entre funções e medidas exponencialmente limitadas é do mesmo tipo.

Lema 1.7. Sejam da uma medida $\gamma$-exponencialmente limitada, isto é, para algum $C_{1}>0$,

$$
\left|v_{\alpha}(t)\right| \leqslant C_{1} e^{\gamma t}
$$

onde $v_{\alpha}$ é a função variação total de $\alpha$. Seja $f$ uma função que satisfaz

$$
|f(t)| \leqslant C_{2} t^{k} e^{\gamma t}
$$

para algum $C_{2}>0$, e $k \geqslant 0$. Então a função $d \alpha * f(t)$ satisfaz

$$
|d \alpha * f(t)| \leqslant C_{1} C_{2} \frac{t^{k+1}}{k+1} e^{\gamma t}
$$


Demonstração. Estimamos

$$
\begin{aligned}
|d \alpha * f(t)| & =\left|\int_{0}^{t} d \alpha(s) f(t-s)\right| \leqslant \int_{0}^{t} d v_{\alpha}(s)|f(t-s)| \\
& \leqslant \int_{0}^{t} C_{1} e^{\gamma s} C_{2}(t-s)^{k} e^{\gamma(t-s)} d s \leqslant C_{1} C_{2} e^{\gamma t} \int_{0}^{t}(t-s)^{k} d s \\
& =C_{1} C_{2} \frac{t^{k+1}}{k+1} e^{\gamma t}
\end{aligned}
$$

o que mostra o resultado.

Corolário 1.8. Sejam da uma medida $\gamma$-exponencialmente limitada e $f$ uma função $\beta$-exponencialmente limitada. Então $d \alpha * f$ é $k$-exponencialmente limitada para todo $k>\max \{\gamma, \beta\}$. 


\section{Capítulo 2}

\section{Equações diferenciais funcionais e equações de renovação}

\subsection{Problema de valor inicial para EDF}

Uma Equação Diferencial Funcional (EDF) linear autônoma é definida pela relação

$$
\frac{d}{d t} M x_{t}=L x_{t}, \quad t \geqslant 0,
$$

onde $L, M: \mathcal{C} \rightarrow \mathbb{C}^{n}$ são operadores lineares contínuos, dados por

$$
L \varphi=\int_{0}^{r} d \eta(\theta) \varphi(-\theta), \quad M \varphi=\varphi(0)-\int_{0}^{r} d \mu(\theta) \varphi(-\theta),
$$

onde $\eta, \mu \in N B V\left([0, r], \mathbb{C}^{n \times n}\right)$ e tomamos $\mu$ contínua em zero para que possamos garantir a existência e unicidade de solução para nossa equação. Para exemplificar, considere a equação diferencial funcional

$$
\frac{d}{d t}[x(t)+C x(t-1)]=A x(t)+B x(t-1), \quad t \geqslant 0
$$

onde $A, B$ e $C$ são matrizes $n \times n$. Tal equação pode ser escrita na forma (2.1) tomando $r=1, \mu(\theta)=0$ se $\theta<1, \mu(\theta)=-C$ para $\theta \geqslant 1$ e $\eta(\theta)=A$ para $0<\theta<1$ e $\eta(\theta)=A+B$ para $\theta \geqslant 1$, então

$$
\begin{aligned}
\frac{d}{d t} M x_{t} & =\frac{d}{d t}\left[x_{t}(0)-\int_{0}^{1} d \mu(\theta) x_{t}(-\theta)\right]=\frac{d}{d t}\left[x_{t}(0)-C x_{t}(-1)\right] \\
& =\frac{d}{d t}[x(t)+C x(t-1)]
\end{aligned}
$$




$$
\begin{aligned}
L x_{t} & =\int_{0}^{r} d \eta(\theta) x_{t}(-\theta)=\int_{0}^{r} d \eta(\theta) x(t-\theta) \\
& =A x(t)+B x(t-1)
\end{aligned}
$$

portanto, de (2.4) e (2.5) temos (2.3).

Um problema de valor inicial para uma Equação Diferencial Funcional linear autônoma é dado por uma EDF da forma (2.1), sujeita à condição inicial

$$
x_{0}=\varphi
$$

ou seja,

$$
\begin{cases}\frac{d}{d t} M x_{t}=L x_{t} & t \geqslant 0 \\ x_{0}=\varphi & \varphi \in \mathcal{C} .\end{cases}
$$

Equações diferenciais da forma (2.1) com $\mu=0$, ou seja,

$$
\dot{x}(t)=L x_{t}
$$

são conhecidas como equações diferenciais funcionais retardadas, (EDFR).

\subsection{Equações de renovação}

No livro de Diekmann et al. [3] foi mostrado que equações diferenciais funcionais retardadas podem ser escritas na forma

$$
x=k * x+f
$$

onde $k$ é uma função de variação limitada e $f$, a função forçante, é contínua. Na tentativa de obter resultados similares para EDF's, estudaremos as equações de renovação e convoluções entre medidas e funções, que serão definidas adiante. Seguem alguns resultados sobre existência, unicidade, dependência contínua e representação de $L^{p}$-soluções da equação integral de Volterra-Stieltjes

$$
z(t)=\int_{0}^{t} d \alpha(s) z(t-s)+f(s)
$$


ou alternativamente,

$$
z=d \alpha * z+f
$$

onde $\alpha \in N B V_{\text {loc }}\left(\mathbb{R}_{+}, \mathbb{C}^{n \times n}\right)$ determina a medida de Borel $d \alpha$, chamada núcleo da equação de renovação $(2.8)$ e $f \in L^{p}\left([0, R], \mathbb{C}^{n}\right)$ é chamada de função forçante.

Observação 2.1. Para $\alpha$ arbitrário, nem sempre podemos esperar a existência e a unicidade para soluções de (2.9). Por exemplo, se $\alpha=\rho \in N B V\left([0, R), \mathbb{C}^{n \times n}\right)$ dada por $\rho(0)=0$ e $\rho(t)=I$ para $t>0$, onde $I$ é a identidade em $\mathbb{C}^{n \times n}$, temos que

$$
d \rho * z(t)=\int_{0}^{t} d \rho(s) z(t-s)=I z(t)=z(t) .
$$

Portanto, a equação de renovação $z=d \rho * z+f$ é equivalente a $f(t) \equiv 0$. Para excluir tais situações, assumimos que

$$
\operatorname{det}\left(\alpha_{0}-I\right) \neq 0, \quad \alpha_{0}=\lim _{t \rightarrow 0^{+}} \alpha(t) .
$$

Temos que $\lim _{t \rightarrow 0^{+}} \operatorname{Var}_{[0, t]}\left(\alpha-\alpha_{0} \rho\right)=0$.

Teorema 2.2. Seja $\alpha \in N B V\left([0, R), \mathbb{C}^{n \times n}\right)$ tal que a condição (2.11) é satisfeita. Então valem:

1. Para todo $f \in \mathcal{C}_{R} \stackrel{\text { def }}{=}\left\{f \in \mathcal{C}\left([0, R], \mathbb{C}^{n}\right): f(0)=0\right\}$ existe uma única solução $z \in \mathcal{C}_{R}$,

$$
z=d \alpha * z+f
$$

em que z depende de $f$ continuamente com respeito à norma do supremo.

2. Para todo $f \in N B V\left([0, R), \mathbb{C}^{n \times n}\right)$, existe uma única solução z de $(2.9)$ em $N B V\left([0, R), \mathbb{C}^{n \times n}\right)$ que depende continuamente de $f$ com respeito a norma $\mathrm{NBV}$.

Demonstração. 1. Seja $A: \mathcal{C}_{R} \mapsto \mathcal{C}_{R}$ dada por

$$
(A x)(t)=d \alpha * x(t)=\int_{0}^{t} d \alpha(s) x(t-s), \quad 0 \leqslant t \leqslant R .
$$

Segue do item 5 da Observação 1.1 que $A$ está bem definido. Afirmamos que o operador $A: \mathcal{C}_{R} \rightarrow \mathcal{C}_{R}$ é linear e limitado. De fato, a linearidade 
de $A$ segue da linearidade da convolução e $A$ é limitado pois $\|A x(t)\|=$ $\|d \alpha * x(t)\| \leqslant \operatorname{Var}_{[0, R)} \alpha \cdot\|x\| \leqslant M .\|x\|$ onde as desigualdades seguem do item 8 da Observação 1.1. Seja $\alpha_{0}=\lim _{t \rightarrow 0^{+}} \alpha(t)$. Tome $\epsilon>0$ e $\gamma>0$ tais que

$$
\operatorname{Var}_{[0, \epsilon]}\left[\alpha-\alpha_{0} \rho\right]+e^{-\gamma \epsilon} \operatorname{Var}_{[0, R)}\left[\alpha-\alpha_{0} \rho\right] \leqslant \frac{1}{2}\left\|\left(I-\alpha_{0}\right)^{-1}\right\|^{-1}
$$

onde $\left\|\left(I-\alpha_{0}\right)^{-1}\right\|$ é a norma usual da matriz $\left(I-\alpha_{0}\right)^{-1}$, isto é,

$$
\left\|\left(I-\alpha_{0}\right)^{-1}\right\|=\sup _{|v|=1}\left|\left(I-\alpha_{0}\right)^{-1} v\right|
$$

onde temos que $|\cdot|$ denota a norma em $\mathbb{C}^{n}$.

Defina em $\mathcal{C}_{R}$ a norma equivalente

$$
\|x\|_{\gamma}=\sup _{0 \leqslant t \leqslant R}|x(t)| e^{-\gamma t}, \quad x \in \mathcal{C}_{R} .
$$

Então, usando (2.10), temos a seguinte estimativa para todo $x \in \mathcal{C}_{R}$ e $t \in$ $[0, R]$

$$
\begin{aligned}
\left|\left(A-\alpha_{0}\right) x(t)\right| e^{-\gamma t}= & \left|\left(d \alpha-\alpha_{0} d \rho\right) * x(t)\right| e^{-\gamma t} \\
= & \left|\int_{0}^{t} d\left(\alpha-\alpha_{0} \rho\right)(s) x(t-s)\right| e^{-\gamma t} \\
\leqslant & \left|\int_{0}^{\epsilon} d\left(\alpha-\alpha_{0} \rho\right)(s) x(t-s)\right| e^{-\gamma t} \\
& +\left|\int_{\epsilon}^{t} d\left(\alpha-\alpha_{0} \rho\right)(s) x(t-s)\right| e^{-\gamma t} \\
\leqslant & \operatorname{Var}_{[0, \epsilon]}\left[\alpha-\alpha_{0} \rho\right] \sup _{t-\epsilon \leqslant \tau \leqslant t}|x(\tau)| e^{-\gamma \tau} \\
& +\operatorname{Var}_{[\epsilon, t)}\left[\alpha-\alpha_{0} \rho\right] \sup _{0 \leqslant \tau \leqslant t-\epsilon}|x(\tau)| e^{-\gamma \tau} \\
\leqslant & \left(\operatorname{Var}_{[0, \epsilon]}\left[\alpha-\alpha_{0} \rho\right]+e^{-\gamma \epsilon} \operatorname{Var}_{[0, R)}\left[\alpha-\alpha_{0} \rho\right]\right)\|x\|_{\gamma} \\
\leqslant & \frac{1}{2}\left\|\left(I-\alpha_{0}\right)^{-1}\right\|^{-1}\|x\|_{\gamma}
\end{aligned}
$$

pois, se $t-\epsilon \leqslant \tau \leqslant t$, então $|x(\tau)| e^{-\gamma t}<|x(\tau)| e^{-\gamma \tau}, \log \sup _{t-\epsilon \leqslant \tau \leqslant t}|x(\tau)| e^{-\gamma t} \leqslant$ $\sup |x(\tau)| e^{-\gamma \tau}=\|x\|_{\gamma}$ e, se $0 \leqslant \tau \leqslant t-\epsilon$, então $e^{-\gamma t}=e^{-\gamma(t-\epsilon)-\gamma \epsilon} \leqslant e^{-\gamma \tau} e^{-\gamma \epsilon}$. Portanto, $\sup _{0 \leqslant \tau \leqslant t-\epsilon}|x(\tau)| e^{-\gamma t} \leqslant e^{-\gamma \epsilon} \sup |x(\tau)| e^{-\gamma \tau}=e^{-\gamma \epsilon}\|x\|_{\gamma}$.

Considere agora a aplicação

$$
x \stackrel{T}{\mapsto} T(x)=\left(I-\alpha_{0}\right)^{-1}\left[A x-\alpha_{0} x+f\right], \quad x \in \mathcal{C}_{R} .
$$


Temos que $T(x) \in \mathcal{C}_{R}$ visto que $A x-\alpha_{0} x+f=d\left(\alpha-\alpha_{0} \rho\right) * x+f$, e ambas parcelas estão em $\mathcal{C}_{R}$. Além disso, $T$ é uma contração em $\mathcal{C}_{R}$ com respeito a norma $\|\cdot\|_{\gamma}$. De fato,

$$
\begin{aligned}
\|T(x)-T(y)\|_{\gamma}= & \sup _{0 \leqslant t \leqslant R}|[T(x)-T(y)](t)| e^{-\gamma t} \\
= & \sup _{0 \leqslant t \leqslant R} \mid\left\{\left(I-\alpha_{0}\right)^{-1}\left[A x-\alpha_{0} x+f\right]\right. \\
& \left.\quad+\left(I-\alpha_{0}\right)^{-1}\left[-A y+\alpha_{0} y-f\right]\right\}(t) \mid e^{-\gamma t} \\
= & \sup _{0 \leqslant t \leqslant R}\left|\left(I-\alpha_{0}\right)^{-1}\left\{\left[A-\alpha_{0}\right](x-y)\right\}(t)\right| e^{-\gamma t} \\
\leqslant & \sup _{0 \leqslant t \leqslant R}\left|\left(I-\alpha_{0}\right)^{-1}\right|\left|\left\{\left[A-\alpha_{0}\right](x-y)\right\}(t)\right| e^{-\gamma t} \\
\leqslant & \sup _{0 \leqslant t \leqslant R}\left|\left(I-\alpha_{0}\right)^{-1}\right| \frac{1}{2}\left|\left(I-\alpha_{0}\right)^{-1}\right|^{-1}\|x-y\|_{\gamma} \\
= & \frac{1}{2}\|x-y\|_{\gamma} .
\end{aligned}
$$

Pelo Teorema do Ponto Fixo de Banach, $T$ tem um único ponto fixo $z \in \mathcal{C}_{R}$. Temos que

$$
\begin{aligned}
z & =z-\alpha_{0} z+\alpha_{0} z=\left(I-\alpha_{0}\right) z+\alpha_{0} z \\
& =\left(I-\alpha_{0}\right) T(z)+\alpha_{0} z=A z-\alpha_{0} z+f+\alpha_{0} z=A z+f \\
& =d \alpha * z+f .
\end{aligned}
$$

Segue o item 1 do teorema.

2. Seja $A x=d \alpha^{T} * x$. Então, da parte 1 aplicada a $\alpha^{T}$, temos que $z=d \alpha^{T} * z+f$ se, e somente se, $z=(I-A)^{-1} f$ e $(I-A)^{-1}$ é linear e contínuo, e portanto $1 \notin \sigma(A)$.

Considere a dualidade $\langle\cdot, \cdot\rangle: \mathcal{C}_{R} \times N B V \rightarrow \mathbb{C}$ dada por

$$
\langle g, \beta\rangle=g^{T} * d \beta(R) .
$$

Afirmamos que $A^{*}: N B V \rightarrow N B V$ é dada por $A^{*} \beta=d \alpha * \beta$. Temos que

$$
\begin{aligned}
\langle A g, \beta\rangle & =\left\langle d \alpha^{T} * g, \beta\right\rangle=\left(d \alpha^{T} * g\right)^{T} * d \beta(R) \\
& =\left(g^{T} * d \alpha\right) * d \beta(R)=g^{T} *(d \alpha * d \beta)(R) \\
& =g^{T} * d(d \alpha * \beta)(R)=\langle g, d \alpha * \beta\rangle .
\end{aligned}
$$

Do fato de $\sigma(A)=\sigma\left(A^{*}\right)$, segue que $1 \notin \sigma\left(A^{*}\right)$, seguindo o resultado. 
Dados $\alpha \in N B V\left([0, R], \mathbb{C}^{n \times n}\right)$ e $f \in L^{p}\left([0, R], \mathbb{C}^{n}\right)$, existem duas maneiras equivalentes de resolver a equação (2.9); ou usando a solução fundamental, ou usando o núcleo resolvente. Apresentaremos as duas a seguir.

\subsubsection{A solução fundamental}

Definição 2.3. Tome $\alpha \in N B V\left([0, R), \mathbb{C}^{n \times n}\right)$ satisfazendo a condição $(2.11)$ na página 15. A única solução $\xi$ de

$$
\xi=d \alpha * \xi+\rho
$$

$\operatorname{com} \rho(0)=0$ e $\rho(t)=I$ para $t>0$ é chamada solução fundamental de (2.9).

O Teorema 2.2 nos garante a existência e a unicidade da solução fundamental. Na verdade, a solução fundamental também resolve a equação

$$
\xi=\xi * d \alpha+\rho
$$

De fato, suponha que $\zeta$ é uma solução de (2.14). Então

$$
\zeta=\zeta * d \rho=\zeta * d[\xi-d \alpha * \xi]=d[\zeta-\zeta * d \alpha] * \xi=d \rho * \xi=\xi
$$

onde a terceira igualdade é verdadeira pois

$$
\begin{aligned}
\zeta * d[\xi-d \alpha * \xi] & =d[\zeta *[\xi-d \alpha * \xi]]=d[(\zeta-\zeta * d \alpha) * \xi] \\
& =d[\zeta-\zeta * d \alpha] * \xi
\end{aligned}
$$

Deste modo, vemos que $\xi^{T}$ é a solução fundamental da equação de renovação transposta

$$
z=d \alpha^{T} * z+f
$$

Lema 2.4. Seja $\alpha \in N B V\left([0, R), \mathbb{C}^{n \times n}\right)$ satisfazendo a condição (2.11) na página 15 e seja $\xi$ a solução fundamental de (2.9). Então, para $f \in L^{p}\left([0, R], \mathbb{C}^{n}\right)$

com $1 \leqslant p \leqslant \infty$, existe uma única solução $z \in L^{p}\left([0, R], \mathbb{C}^{n}\right)$ de $(2.9)$, isto é, $z=d \alpha * z+f$. Esta solução é dada por

$$
z=d \xi * f
$$

e depende continuamente de $f$ com respeito à norma $L^{p}$. 
Demonstração. Seja $x \in L^{p}\left([0, R], \mathbb{C}^{n}\right)$ dada por (2.15). Então, por (2.13), temos que

$$
\begin{aligned}
x=d \xi * f=d(d \alpha * \xi+\rho) * f & =(d \alpha * d \xi+d \rho) * f=d \alpha *(d \xi * f)+d \rho * f \\
& =d \alpha * x+f .
\end{aligned}
$$

Reciprocamente, seja $x$ uma solução de (2.9). Então, por (2.14),

$$
x=d \rho * x=d(\xi-\xi * d \alpha) * x=\xi * d[x-d \alpha * x]=d \xi * f
$$

o que prova a existência e unicidade e que $x=d \xi * f$. A dependência contínua segue de (2.15) e de (1.6).

\subsubsection{Núcleo resolvente}

Definição 2.5. Tome $\alpha \in N B V\left([0, R), \mathbb{C}^{n \times n}\right)$ satisfazendo a condição $(2.11)$ na página 15. A única solução $\zeta$ de

$$
\zeta=\zeta * d \alpha+\alpha
$$

é chamada de núcleo resolvente de (2.9).

Analogamente ao caso da solução fundamental, temos que o núcleo resolvente também resolve

$$
\zeta=d \alpha * \zeta+\alpha
$$

De fato, se $\delta$ é solução de (2.17), então

$$
\begin{aligned}
\delta & =d \alpha * \delta+\alpha=d[\zeta-\zeta * d \alpha] * \delta+\alpha=d \zeta * \delta-d \zeta * d \alpha * \delta+\alpha \\
& =\zeta * d \delta-d \zeta * \alpha * d \delta+\alpha=\zeta * d \delta-\zeta * d \alpha * d \delta+\alpha \\
& =\zeta *[d \delta-d \alpha * d \delta]+\alpha=\zeta * d[\delta-d \alpha * \delta]+\alpha \\
& =\zeta * d \alpha+\alpha=\zeta
\end{aligned}
$$

e, portanto, $\zeta^{T}$ é o núcleo resolvente da equação de renovação transposta

$$
z=d \alpha^{T} * z+f
$$


Lema 2.6. Seja $\alpha \in N B V\left([0, R), \mathbb{C}^{n \times n}\right)$ satisfazendo a condição (2.11) na página 15 e seja $\zeta$ o núcleo resolvente de (2.8). Então, para $f \in L^{p}\left([0, R], \mathbb{C}^{n}\right)$ com $1 \leqslant p \leqslant \infty$, existe uma única solução $z \in L^{p}\left([0, R], \mathbb{C}^{n}\right)$ de $(2.9)$, isto é, $z=d \alpha * z+f$. Esta solução é dada por

$$
z=d \zeta * f+f
$$

e depende continuamente de $f$ com respeito a norma $L^{p}$. Além disso, se $f \in \mathcal{C}\left([0, R], \mathbb{C}^{n}\right)$ e $f(0)=0$, então a solução z satisfaz z $\in \mathcal{C}\left([0, R], \mathbb{C}^{n}\right)$ e $z(0)=0$. Se $f \in N B V\left([0, R), \mathbb{C}^{n}\right)$, então a solução z também satisfaz $z \in N B V\left([0, R), \mathbb{C}^{n}\right)$.

Demonstração. De (2.9) temos que

$$
z=d \alpha * z+f
$$

Se aplicarmos a convolução por $d \zeta$ a ambos os lados desta equação, obtemos

$$
\begin{aligned}
d \zeta * z & =d \zeta *(d \alpha * z)+d \zeta * f \\
& =(d \zeta * d \alpha) * z+d \zeta * f \\
& =d(\zeta * d \alpha) * z+d \zeta * f \\
& =d(\zeta-\alpha) * z+d \zeta * f \\
& =d \zeta * z-d \alpha * z+d \zeta * f
\end{aligned}
$$

Portanto, temos que

$$
d \zeta * f=d \alpha * z
$$

Agora, substituindo (2.19) em (2.9), temos (2.18). A dependência contínua de $z$ com respeito a $f$ segue da solução explícita (2.18) e da desigualdade (1.6). Do item 5 da Observação 1.1, segue que, se $f \in \mathcal{C}\left([0, R], \mathbb{C}^{n}\right)$ e $f(0)=0$, então a solução $z \in \mathcal{C}\left([0, R], \mathbb{C}^{n}\right)$ e $z(0)=0$ e, se $f \in N B V\left([0, R), \mathbb{C}^{n}\right)$, então a solução $z \in N B V\left([0, R), \mathbb{C}^{n}\right)$.

Teorema 2.7. Seja $\alpha \in N B V_{\text {loc }}\left(\mathbb{R}_{+}, \mathbb{C}^{n \times n}\right)$ satisfazendo a condição (2.11). Então existe um único $\zeta \in N B V_{\text {loc }}\left(\mathbb{R}_{+}, \mathbb{C}^{n \times n}\right)$ tal que

$$
\zeta(t)=[\zeta * d \alpha](t)+\alpha(t), \quad t \geqslant 0
$$


Demonstração. Se $\beta \in N B V\left([0, R), \mathbb{C}^{n \times n}\right)$, temos que $\beta(t)=\beta(R-)$ para $t \geqslant R$. Tomando $R>0$, definimos $\alpha_{R} \in N B V\left([0, R), \mathbb{C}^{n \times n}\right)$ por $\alpha_{R}(t)=$ $\alpha(t)$ para $0 \leqslant t<R$ e $\alpha_{R}(t)=\alpha(R-)$ para $t \geqslant R$. Denotamos por $\zeta_{R} \in N B V\left([0, R), \mathbb{C}^{n \times n}\right)$ o núcleo resolvente da equação de renovação (2.8), onde $\alpha$ é substituido por $\alpha_{R}$. Assim

$$
\zeta_{R}(t)=d \alpha_{R} * \zeta_{R}(t)+\alpha_{R}(t), \quad 0 \leqslant t \leqslant R,
$$

o que prova o resultado.

Corolário 2.8. Seja $\alpha \in N B V_{\text {loc }}\left(\mathbb{R}_{+}, \mathbb{C}^{n \times n}\right)$ satisfazendo a condição (2.11). Para todas as funções $f:[0, \infty) \rightarrow \mathbb{C}^{n}$ que satisfaçam uma das seguintes propriedades

1. A restrição de $f$ a intervalos $[0, R]$ pertence a $L^{p}\left([0, R], \mathbb{C}^{n}\right)$, para $R>$ 0 e $1 \leqslant p \leqslant \infty$

2. $f$ pertence a $N B V_{\text {loc }}\left(\mathbb{R}_{+}, \mathbb{C}^{n}\right)$;

3. A restrição de $f$ a intervalos $[0, R]$ pertence a $\mathcal{C}\left([0, R], \mathbb{C}^{n}\right)$, para $R>0$ e $f(0)=0$.

existe uma única solução z de (2.9), isto é

$$
z(t)=[d \alpha * z](t)+f(t)
$$

tal que z tem as mesmas propriedades de $f$. Além disso, z é dada explicitamente pela fórmula

$$
z(t)=[d \zeta * f](t)+f(t), \quad t \geqslant 0
$$

Demonstração. Segue do Lema 2.6 extendendo as conclusões para funções definidas em $[0, \infty)$. O núcleo resolvente é encontrado a partir do Teorema 2.7 . 


\subsection{Representação de uma EDF como uma equação de renovação}

Estabeleceremos aqui uma equivalência entre equações diferenciais funcionais e uma classe de equações de renovação. Consideramos, como na seção anterior, o problema de valor inicial em EDF

$$
\begin{cases}\frac{d}{d t} M x_{t}=L x_{t} & t \geqslant 0 \\ x_{0}=\varphi & \varphi \in \mathcal{C}\end{cases}
$$

onde $M, L: \mathcal{C} \rightarrow \mathbb{C}^{n}$ são dados por

$$
L \varphi=\int_{0}^{r} d \eta(\theta) \varphi(-\theta), \quad M \varphi=\varphi(0)-\int_{0}^{r} d \mu(\theta) \varphi(-\theta) .
$$

e $\mu, \eta \in N B V\left([0, r], \mathbb{C}^{n \times n}\right)$ com $\mu$ contínua em 0 . Iniciamos nosso estudo com o seguinte resultado.

Lema 2.9. O problema de valor inicial (2.23) é equivalente à seguinte equação de renovação

$$
x(t)=d k * x(t)+F \varphi(t) \quad t \geqslant 0,
$$

escrita na forma integral como

$$
x(t)=\int_{0}^{t}[d k(\theta) x(t-\theta)+F \varphi(t), \quad t \geqslant 0
$$

onde $k$ é dado por

$$
k(\theta)=\mu(\theta)+\int_{0}^{\theta} \eta(s) d s
$$

e $F: \mathcal{C} \rightarrow L^{\infty}$, definida por

$$
F \varphi(t)=M \varphi+\int_{t}^{r} d \mu(\theta) \varphi(t-\theta)+\int_{0}^{t}\left[\int_{s}^{r} d \eta(\theta) \varphi(s-\theta)\right] d s, \quad t \geqslant 0
$$

aplica a condição inicial $\varphi \in \mathcal{C}$ na função forçante correspondente da equação de renovação (2.25). Para $\varphi \in \mathcal{C}$, temos que $F \varphi(\cdot)$ é constante em $[r, \infty)$, $F \varphi(0)=\varphi(0)$ e $F \varphi+\mu \cdot \varphi(0)$ é contínua. 
Demonstração. Integrando o sistema (2.23), obtemos

$$
M x_{t}-M \varphi=\int_{0}^{t}\left[\int_{0}^{r} d \eta(\theta) x(s-\theta)\right] d s .
$$

Agora, para qualquer $\xi \in N B V\left([0, r], \mathbb{C}^{n \times n}\right)$ e para $0<t \leqslant r$, temos

$$
\begin{aligned}
\int_{0}^{r} d \xi(\theta) x(t-\theta) & =\int_{0}^{t} d \xi(\theta) x(t-\theta)+\int_{t}^{r} d \xi(\theta) x(t-\theta) \\
& =\int_{0}^{t} d \xi(\theta) x(t-\theta)+\int_{t}^{r} d \xi(\theta) \varphi(t-\theta)
\end{aligned}
$$

pois para $t \leqslant \theta \leqslant r, t-\theta \leqslant 0$ e $x(t-\theta)=\varphi(t-\theta)$. Para $t>r$, usando o fato de que para $\theta \notin[0, r]$, a variação de $\xi$ se anula em $(r, t]$, vemos que

$$
\begin{aligned}
\int_{t}^{r} d \xi(\theta) \varphi(t-\theta) & =0 \\
\int_{0}^{t} d \xi(\theta) x(t-\theta) & =\int_{0}^{r} d \xi(\theta) x(t-\theta) .
\end{aligned}
$$

Portanto, para $t>0$ e para todo $\xi \in N B V\left([0, r], \mathbb{C}^{n \times n}\right)$, escrevemos

$$
\int_{0}^{r} d \xi(\theta) x(t-\theta)=\int_{0}^{t} d \xi(\theta) x(t-\theta)+\int_{t}^{r} d \xi(\theta) \varphi(t-\theta) .
$$

Aplicando (2.31) em (2.29), para $t>0$, temos que

$$
\begin{aligned}
x(t)= & \int_{0}^{r} d \mu(\theta) x(t-\theta)+M \varphi+\int_{0}^{t}\left[\int_{0}^{r} d \eta(\theta) x(s-\theta)\right] d s \\
= & \int_{0}^{t} d \mu(\theta) x(t-\theta)+\int_{t}^{r} d \mu(\theta) \varphi(t-\theta)+M \varphi \\
& \quad+\int_{0}^{t}\left[\int_{0}^{s} d \eta(\theta) x(s-\theta)+\int_{s}^{r} d \eta(\theta) \varphi(s-\theta)\right] d s \\
= & \int_{0}^{t} d \mu(\theta) x(t-\theta)+\int_{0}^{t}\left[\int_{0}^{s} d \eta(\theta) x(s-\theta)\right] d s \\
& \quad+M \varphi+\int_{t}^{r} d \mu(\theta) \varphi(t-\theta)+\int_{0}^{t}\left[\int_{s}^{r} d \eta(\theta) \varphi(s-\theta)\right] d s .
\end{aligned}
$$

Aplicando o Teorema de Fubini na segunda integral e definindo $F \varphi(t)$ como em (2.28), que contém explicitamente o dado inicial $\varphi$, temos

$x(t)=\int_{0}^{t} d \mu(\theta) x(t-\theta)+\int_{0}^{t}\left[\int_{0}^{s} d \eta(\theta) x(s-\theta)\right] d s+F \varphi(t)$ 


$$
\begin{aligned}
= & \int_{0}^{t} d \mu(\theta) x(t-\theta)+\int_{0}^{t} d \eta(\theta)\left[\int_{\theta}^{t} x(s-\theta) d s\right]+F \varphi(t) \\
= & \int_{0}^{t} d \mu(\theta) x(t-\theta)+\int_{0}^{t} d \eta(\theta)\left[\int_{0}^{t-\theta} x(s) d s\right]+F \varphi(t) \\
= & \int_{0}^{t} d \mu(\theta) x(t-\theta)+\left.\eta(\theta)\left[\int_{0}^{t-\theta} x(s) d s\right]\right|_{\theta=0} ^{t}+\int_{0}^{t} \eta(\theta) x(t-\theta) d \theta+F \varphi(t) \\
= & \int_{0}^{t} d \mu(\theta) x(t-\theta)+\int_{0}^{t} \eta(\theta) x(t-\theta) d \theta+F \varphi(t) \\
= & \int_{0}^{t}[d \mu(\theta)+\eta(\theta) d \theta] x(t-\theta)+F \varphi(t) \\
= & \int_{0}^{t} d k(\theta) x(t-\theta)+F \varphi(t)=d k * x(t)+F \varphi(t) .
\end{aligned}
$$

Temos que

$$
\begin{aligned}
F \varphi(0) & =M \varphi+\int_{0}^{r} d \mu(\theta) \varphi(-\theta) \\
& =\varphi(0) .
\end{aligned}
$$

Como $\mu$ é contínua em $\theta=0$, segue que $k$ também o é. Logo (2.25) também é verdadeira para $t=0$. Também podemos mostrar que $F \varphi(t)$ é constante para $t \geqslant r$, uma vez que as variações de $\mu$ e $\eta$ são nulas para $\theta \geqslant r$.

Mostremos agora a continuidade de $F \varphi+\mu \cdot \varphi(0)$. O único termo não contínuo em (2.28) é o segundo. Então, a extensão de $\varphi$ de $[-r, 0]$ a $[-r, \infty)$ definida por $\varphi(\theta)=\varphi(0)$, se $\theta \geqslant 0$, é contínua. Além disso, a aplicação $t \stackrel{T}{\mapsto} \varphi_{t}$ é contínua. Como $\psi \stackrel{S}{\mapsto} \int_{0}^{r} d \mu(\theta) \psi(-\theta)$ também é uma aplicação contínua, segue que a composição

$$
\begin{aligned}
t \stackrel{S \circ T}{\longmapsto} \int_{0}^{r} d \mu(\theta) \varphi_{t}(-\theta) & =\int_{0}^{t} d \mu(\theta) \varphi(t-\theta)+\int_{t}^{r} d \mu(\theta) \varphi(t-\theta) \\
& =\int_{0}^{t} d \mu(\theta) \varphi(0)+\int_{t}^{r} d \mu(\theta) \varphi(t-\theta) \\
& =\mu(t) \varphi(0)+\int_{t}^{r} d \mu(\theta) \varphi(t-\theta)
\end{aligned}
$$

é contínua. Portanto,

$$
\int_{t}^{r} d \mu(\theta) \varphi(t-\theta)=\int_{0}^{r} d \mu(\theta) \varphi_{t}(-\theta)-\mu(t) \cdot \varphi(0)
$$

de onde segue que $F \varphi+\mu \cdot \varphi(0)$ é contínua. 
Teorema 2.10. A solução $x(t)$ do PVI (2.23) é contínua. Além disso $x(t)$ é dada por

$$
x=d \zeta * F \varphi+F \varphi
$$

onde $\zeta$ é o núcleo resolvente de $k$ (cf. Def. 2.5 e Teo. 2.7), e $k$ é dado por $(2.27)$.

Demonstração. Podemos reescrever a equação de renovação (2.25) na forma

$$
y=d k * y+F_{0}(\varphi)
$$

onde $y=x-\varphi(0)$ e com

$$
F_{0}(\varphi) \stackrel{\text { def }}{=} F \varphi-\varphi(0)+k \cdot \varphi(0)
$$

da seguinte forma:

$$
\begin{gathered}
x=d k * x+F \varphi \\
x-\varphi(0)=d k * x+F \varphi-\varphi(0) \\
x-\varphi(0)=d k *[x-\varphi(0)]+F \varphi-\varphi(0)+d k * \varphi(0) \\
y=d k * y+\underbrace{F \varphi-\varphi(0)+k \cdot \varphi(0)}_{\stackrel{\text { def }}{=} F_{0}(\varphi)} .
\end{gathered}
$$

Das propriedades de $F \varphi$ do Lema 2.9, segue que $F_{0}(\varphi)(\cdot)$ é contínua em $[0, \infty)$ e $F_{0}(\varphi)(0)=0$. Do Teorema 2.7 , na página 20 , com $\alpha=k$, onde $k$ é dado por (2.27), encontramos o único núcleo resolvente $\zeta$. Usando a forma explícita da solução, dada pelo Corolário 2.8, na página 21, temos

$$
y=d \zeta * F_{0}(\varphi)+F_{0}(\varphi)
$$

como uma representação alternativa da solução do PVI (2.23) por meio da solução de uma equação de renovação em termos do espaço $\left\{f \in \mathcal{C}\left([0, \infty), \mathbb{C}^{n}\right)\right.$; $f(0)=0\}$. Destas considerações, segue que $y=x-\varphi(0)$ é contínua e $y(0)=0$. Isto nos dá que as soluções $x(t)$ de (2.23) são contínuas e $x(0)=\varphi(0)$.

Além disso, de (2.35) e (2.34), e usando $d \zeta * k=\zeta-k$, temos

$$
y=d \zeta * F_{0}(\varphi)+F_{0}(\varphi)
$$




$$
\begin{gathered}
x-\varphi(0)=d \zeta *(F \varphi-\varphi(0)+k \cdot \varphi(0))+F \varphi-\varphi(0)+k \cdot \varphi(0) \\
x=d \zeta * F \varphi-d \zeta * \varphi(0)+d \zeta * k \cdot \varphi(0)+F \varphi+k \cdot \varphi(0) \\
x=d \zeta * F \varphi-\zeta \cdot \varphi(0)+(\zeta-k) \cdot \varphi(0)+F \varphi+k \cdot \varphi(0) \\
x=d \zeta * F \varphi+F \varphi .
\end{gathered}
$$

donde obtemos (2.32).

Finalizamos esta seção dando estimativas da limitação exponencial tanto do núcleo resolvente quanto da solução. Estas estimativas garantem que podemos aplicar resultados das transformadas de Laplace-Stieltjes no estudo das soluções das EDF.

\section{Limitação exponencial das soluções de equações de renovação}

Lema 2.11. O núcleo $k$ dado por (2.27), isto é,

$$
k(\theta)=\mu(\theta)+\int_{0}^{\theta} \eta(s) d s,
$$

é $\epsilon$-exponencialmente limitado para todo $\epsilon>0$. Além disso, temos

$$
\left|v_{k}\right|<C t
$$

para $C>0$ suficientemente grande, onde $v_{k}$ é a função variação total de $k$.

Demonstração. Como $\mu$ e $\eta$ pertencem a $N B V\left([0, r], \mathbb{C}^{n \times n}\right)$, temos que $\eta$ é constante a partir de $\theta \geqslant r$ e

$$
k(\theta)=k(r)+(\theta-r) \eta(r)
$$

Disto seguem todas as afirmações a respeito de $k$.

Teorema 2.12. O núcleo resolvente $\zeta$ de $k$, onde $k$ é dado por (2.27), pertence a $N B V_{\text {loc }}\left(\mathbb{R}_{+}, \mathbb{C}^{n \times n}\right)$ e é $\gamma$-exponencialmente limitado, para algum $\gamma$ suficientemente grande. Além disso, $\zeta$ é dado pela série

$$
\zeta=k+d k * k+d k * d k * k+\cdots
$$


Demonstração. Dos Lemas 1.7 e 2.11, temos que

$$
\begin{gathered}
|k(t)| \leqslant C t \\
|d k * k(t)| \leqslant C^{2} \frac{t^{2}}{2 !} \\
|d k * d k * k(t)| \leqslant C^{3} \frac{t^{3}}{3 !}
\end{gathered}
$$

e de forma geral,

$$
|\underbrace{d k * d k * \cdots * d k * k(t)}_{n-1 \text { convoluções }}| \leqslant C^{n} \frac{t^{n}}{n !} .
$$

Desta forma, podemos estimar a série

$$
|k+d k * k+d k * d k * k+\cdots| \leqslant \sum_{n=1}^{\infty} \frac{(C t)^{n}}{n !}=e^{C t}
$$

Como as mesmas estimativas (2.38) e (2.39) valem para a variação total de $k$, como cada parcela é $N B V_{\text {loc }}$ (cf. Corolário 2.8) e como $N B V_{\text {loc }}$ é completo, segue que o limite da série é uma função localmente de variação limitada. Defina $\zeta$ como o valor da série. Destas discussões, vem que

$$
v_{\zeta}(t) \leqslant e^{C t}
$$

isto é, $\zeta$ é $C$-exponencialmente limitada. Por simples substituição, é fácil ver que $\zeta$ definida como acima é o núcleo resolvente de $k$, isto é,

$$
\zeta=d k * \zeta+k
$$

Isto nos prova o resultado.

Da representação (2.32) para a solução do PVI (2.23), segue imediatamente o seguinte fato.

Corolário 2.13. A solução x de (2.23), dada por (2.32), é $\gamma$-exponencialmente limitada para $\gamma$ suficientemente grande. 


\subsection{O espaço das funções forçantes}

Nesta seção, descreveremos o espaço das funções forçantes através da representação de EDF's como equações de renovação (2.25).

A partir do item 5 da Observação 1.1, para $\alpha \in N B V\left([0, r], \mathbb{C}^{n \times n}\right)$, temos que a aplicação $f \mapsto d \alpha * f$ leva o espaço $\left\{f \in \mathcal{C}\left([0, r], \mathbb{C}^{n}\right): f(0)=0\right\}$ em si mesmo, mas o espaço $\mathcal{C}$ não é necessariamente aplicado em si mesmo por este operador. Portanto, a correspondência biunívoca entre a função forçante e a solução da equação de renovação é fechada no espaço $\left\{f \in \mathcal{C}\left([0, r], \mathbb{C}^{n}\right)\right.$ : $f(0)=0\}$, mas não o é em $\mathcal{C}\left([0, r], \mathbb{C}^{n}\right)$. As mesmas afirmações são válidas para os espaços $\left\{f \in \mathcal{C}\left([0, \infty), \mathbb{C}^{n}\right): f(0)=0\right\}$ e $\mathcal{C}\left([0, \infty), \mathbb{C}^{n}\right)$. Para EDF's retardadas, isto é, quando $\mu \equiv 0$, encontramos em Hale \& Verduyn Lunel [5] que $\left\{f \in \mathcal{C}\left([0, \infty), C^{n}\right): f(t)=f(r)\right.$, para $\left.t \geqslant r\right\}$ é uma escolha comum para o espaço das funções forçantes da representação desta classe de EDF's como equações de renovação. Observamos que para EDFR, a restrição $f(0)=0$

não é necessária, pois $k(\theta)=\int_{0}^{\theta} \eta(\tau) d \tau$, que é contínua. Do item 5 da Observação 1.1 , vem que os espaços $\mathcal{C}\left([0, r], \mathbb{C}^{n}\right)$ e $\mathcal{C}\left([0, \infty), \mathbb{C}^{n}\right)$ são fechados com respeito à aplicação $f \mapsto d k * f$.

Definição 2.14. O espaço $\mathcal{F}$ das funções forçantes $f$ da equação de renovação

$$
x=d k * x+f
$$

onde $k$ é como em (2.27) é definido como o conjunto das funções $f:[0, \infty) \mapsto$ $\mathbb{C}^{n}$ tais que $f+\mu \cdot f(0)$ é contínua e $f(t)=f(r)$ para $t \geqslant r$. Pelo Lema 2.9, para cada $\varphi \in \mathcal{C}$, temos que $F \varphi \in \mathcal{F}$.

Com esta definição, podemos ver com clareza as afirmações acima acerca de EDFR's, ou seja, segue desta definição que, como $\mu \equiv 0$, então o espaço das funções forçantes para EDFR será dado por $\mathcal{F}=\left\{f \in \mathcal{C}\left([0, \infty) \mathbb{C}^{n}\right)\right.$ : $f(t)=f(r)$ para $t \geqslant r\}$.

Temos que $\mathcal{F}$ é um espaço linear e podemos definir uma norma $\|\cdot\|_{\mathcal{F}}$ em $\mathcal{F}$ por

$$
\|f\|_{\mathcal{F}}=\|f+\mu \cdot f(0)\|_{\mathcal{C}\left([0, \infty), \mathbb{C}^{n}\right)}
$$

onde $\|\cdot\|$ é a norma do supremo, de modo que o espaço é completo. 
Lema 2.15. Para cada $f \in \mathcal{F}$, existe uma única solução $x$ definida em $[0, \infty)$ de (2.40) tal que $x$ é contínua em $[0, \infty)$ e $x(0)=f(0)$.

Demonstração. Temos as seguintes equações equivalentes

$$
\begin{gathered}
x=d k * x+f \\
x-f(0)=d k * x+f-f(0) \\
x-f(0)=d k *[x-f(0)]+f-f(0)+d k * f(0) \\
x-f(0)=d k *[x-f(0)]+f-f(0)+k \cdot f(0) \\
x-f(0)=d k *[x-f(0)]+f-f(0)+\left[\mu+\int \eta\right] \cdot f(0) .
\end{gathered}
$$

Como $f+k \cdot f(0)-f(0)$ é contínua e se anula em 0 , pelo Corolário $2.8, x-f(0)$ é contínua e $x(0)-f(0)=0, \log o x(t)$ é contínua e $x(0)=f(0)$.

\subsection{Resolução de EDF por transformadas de Laplace}

Nas seções anteriores, vimos que a equação de renovação

$$
x=d k * x+f
$$

onde $f \in \mathcal{F}$, está relacionada com o problema de valor inicial (2.23) pelos Lema 2.9 e Teorema 2.10. Sua solução é dada por

$$
x=d \zeta * f+f
$$

onde $\zeta$ é o núcleo resolvente de (2.41) ( $\zeta$ é uma função $N B V$ ). Do Teorema 2.12 segue que $\zeta \in N B V_{\text {loc }}$ e é exponencialmente limitada. Do Corolário 2.13, segue que $x$ é $\gamma$-exponencialmente limitada. Portanto, podemos aplicar a transformada de Laplace em (2.41) para obter, para Re $z$ suficientemente grande, a equação

$$
\mathcal{L}(x)(z)=\mathcal{L}(d k)(z) \mathcal{L}(x)(z)+\mathcal{L}(f)(z)
$$

que torna-se

$$
\begin{aligned}
\mathcal{L}(x)(z) & =(I-\mathcal{L}(d k)(z))^{-1} \mathcal{L}(f)(z) \\
& =(z I-z \mathcal{L}(d k)(z))^{-1} z \mathcal{L}(f)(z)
\end{aligned}
$$




$$
=\Delta(z)^{-1}(f(0)+\mathcal{L}(d f)(z)) .
$$

Note que $\mathcal{L}(d f)(z)$ está bem definida pois $f$ é soma de uma função contínua com uma função localmente de variação limitada e $\Delta(z)$ é definido por

$$
\begin{aligned}
\Delta(z) & =z(I-\mathcal{L}(d k)(z)) \\
& =(z I-z \mathcal{L}(d \mu)(z)-z \mathcal{L}(\eta)(z))=(z I-z \mathcal{L}(d \mu)(z)-\mathcal{L}(d \eta)(z)) \\
& =z\left[I-\int_{0}^{r} e^{-z t} d \mu(t)\right]-\int_{0}^{r} e^{-z t} d \eta(t) .
\end{aligned}
$$

Como $z \mapsto \Delta(z)$ e $z \mapsto \int_{0}^{r} e^{-z t} d f(t)$ são funções inteiras, então o lado direito de (2.44) é uma função meromorfa com, possivelmente, pólos nas raízes da equação característica

$$
\operatorname{det} \Delta(z)=0
$$

Mostremos que existe um semi-plano que não possui raízes de $\operatorname{det} \Delta(z)$.

Lema 2.16. As raízes de (2.46) estão localizadas em um semi-plano esquerdo $\left\{z \in \mathbb{C}: \operatorname{Re} z<\gamma_{0}\right\}$ para algum $\gamma_{0} \in \mathbb{R}$.

Demonstração. Mostremos que, quando $\operatorname{Re} z \rightarrow \infty$, então

$$
\int_{0}^{r} e^{-z \theta} d \mu(\theta) \rightarrow 0 \quad \text { e } \quad \int_{0}^{r} e^{-z \theta} d \eta(\theta) \rightarrow \eta(0-) .
$$

Para isto, seja $\epsilon>0$ dado. Como $\mu$ é contínua em 0 , existe $\delta>0$ tal que a variação de $\mu$ em $[0, \delta]$ é menor que $\frac{\epsilon}{2}$. Tome $N>0$ tal que $e^{-\delta N} \operatorname{Var}_{[\delta, r]} \mu<$ $\frac{\epsilon}{2}$. Então, para $z$ tal que $\operatorname{Re} z>N$, estimamos

$$
\begin{aligned}
\left\|\int_{0}^{r} e^{-z \theta} d \mu(\theta)\right\| & =\left\|\int_{0}^{\delta} e^{-z \theta} d \mu(\theta)+\int_{\delta}^{r} e^{-z \theta} d \mu(\theta)\right\| \\
& \leqslant\left\|\int_{0}^{\delta} e^{-z \theta} d \mu(\theta)\right\|+\left\|\int_{\delta}^{r} e^{-z \theta} d \mu(\theta)\right\| \\
& \leqslant \max _{\theta \in[0, \delta]}\left|e^{-z \theta}\right| \operatorname{Var}_{[0, \delta]} \mu+\max _{\theta \in[\delta, r]}\left|e^{-z \theta}\right| \operatorname{Var}_{[\delta, r]} \mu \\
& \leqslant \operatorname{Var}_{[0, \delta]} \mu+e^{-\delta N} \operatorname{Var}_{[\delta, r]} \mu<\frac{\epsilon}{2}+\frac{\epsilon}{2}=\epsilon .
\end{aligned}
$$

Concluímos a demonstração argumentando da mesma forma para $\bar{\eta}=\eta-$ $\eta(0-)$, que é contínua em zero. Então temos que, quando Re $z$ é suficientemente grande, $\Delta(z)=z\left(I-\int_{0}^{r} e^{-z \theta} d \mu(\theta)\right)-\int_{0}^{r} e^{-z \theta} d \eta(\theta)$ se aproxima de $z I-\eta(0-)$ e, consequentemente, é não-singular. 
Podemos inverter a representação da transformada de Laplace da solução para obter uma caracterização para a solução.

Teorema 2.17. Seja $k$ dado por $k(\theta)=\mu(\theta)+\int_{0}^{\theta} \eta(s) d s$, onde $\mu$ e $\eta$ são funções $N B V$ e $\mu$ é contínua em $\theta=0$. Para $f \in \mathcal{F}$, a solução $x$ da equação de renovação

$$
x=d k * x+f
$$

admite, para $t>0$, a representação

$$
x(t)=\frac{1}{2 \pi i} \int_{(\gamma)} e^{z t} \Delta(z)^{-1}\left(f(0)+\int_{0}^{r} e^{-z \theta} d f(\theta)\right) d z
$$

para $\gamma$ suficientemente grande.

Demonstração. Pelo Lema 2.16, temos que $\Delta(z)$ é não-singular para $\operatorname{Re} z$ suficientemente grande. Como $x$ é contínua e exponencialmente limitada, temos então, pela fórmula de inversão (1.12), que

$$
x(t)=\lim _{\omega \rightarrow \infty} \frac{1}{2 \pi i} \int_{\gamma-i \omega}^{\gamma+i \omega} e^{z t} \mathcal{L}(x)(z) d z \stackrel{\text { def }}{=} \frac{1}{2 \pi i} \int_{(\gamma)} e^{z t} \mathcal{L}(x)(z) d z .
$$

Mas por (2.44) temos que

$$
\mathcal{L}(x)(z)=\Delta(z)^{-1}(f(0)+\mathcal{L}(d f)(z))
$$

de onde segue que

$$
x(t)=\frac{1}{2 \pi i} \int_{(\gamma)} e^{z t} \Delta(z)^{-1}\left(f(0)+\int_{0}^{\infty} e^{-z \theta} d f(\theta)\right) d z .
$$

O seguinte exemplo nos mostra, utilizando as técnicas estudadas, um método para calcular o núcleo resolvente.

Exemplo 2.18. Considere a seguinte EDF

$$
\dot{x}(t)=A x(t-1)
$$

que pode ser escrita na forma $\frac{d}{d t} M x_{t}=L x_{t}$ se tomarmos $r=1, \mu \equiv 0 \mathrm{e}$ $\eta(\theta)=A h_{1}(\theta)$. 
Deste modo, utilizando o Lema (2.9), temos que o núcleo da equação de renovação correspondente a essa EDF é dado por

$$
k(\theta)=h_{1}(\theta) A(\theta-1)
$$

e então, aplicando a transformada de Laplace-Stieltjes, obtemos que

$$
\mathcal{L}(k)(z)=\frac{A e^{-z}}{z^{2}}
$$

Como o núcleo resolvente $\zeta$ é dado pela equação $\zeta=d \zeta * k+k$, concluímos que

$$
\mathcal{L}(\zeta)(z)=\frac{A e^{-z}}{z\left(z-A e^{-z}\right)}
$$

e, portanto, podemos obter o núcleo resolvente, aplicando a transformada de Laplace-Stieltjes inversa, ou seja,

$$
\zeta(t)=\mathcal{L}^{-1}\left[\frac{A e^{-s}}{s\left(s-A e^{-s}\right)}\right] .
$$

Da representação (2.49) da solução de (2.48), conseguimos estudar o comportamento assintótico das soluções, porém, antes, é necessário estudar algumas estimativas relacionadas a $\Delta(z)$. 


\section{Capítulo 3}

\section{Comportamento assintótico}

\subsection{Estimativas para $\Delta(z)$ e quantidades relacionadas}

Nesta seção estudaremos alguns resultados relacionados com a localização das raízes e algumas estimativas da matriz característica $\Delta(z)$, dada em (2.45), além de seu determinante e sua inversa. A partir desses resultados, estudaremos o comportamento assintótico das soluções. Podemos escrever $\Delta(z)$ como

$$
\Delta(z)=z \Delta_{0}(z)-\int_{0}^{r} e^{-z \theta} d \eta(\theta)
$$

onde

$$
\Delta_{0}(z)=I-\int_{0}^{r} e^{-z \theta} d \mu(\theta) .
$$

É possível que existam infinitas raízes da equação característica (2.46) em uma faixa vertical do plano complexo $\{z \in \mathbb{C}: a<\operatorname{Re} z<b\}$. Um exemplo é considerar a equação característica da seguinte EDF escalar

$$
\dot{x}(t)-\dot{x}(t-1)=0
$$

dada por

$$
\Psi(z)=z\left(1-e^{-z}\right)
$$

e tem todas as suas raízes da forma $2 k \pi i \operatorname{com} k \in \mathbb{Z}$ e, portanto, estão sobre a reta $\operatorname{Re} z=0$. Isto é uma complicação extra para equações neutras que não é observado em equações diferenciais retardadas. Veja, por exemplo, Teorema I.4.4 de Diekmann et al. [3]. 
Para que possamos controlar o comportamento de $|\Delta(z)|$ quando $|z| \rightarrow$ $\infty$, impomos a seguinte hipótese sobre o núcleo $\mu$ :

(J) As entradas $\mu_{i j}$ de $\mu$ têm um salto antes de se tornarem constantes, isto é, existe $t_{i j} \operatorname{com} \mu_{i j}\left(t_{i j}-\right) \neq \mu_{i j}\left(t_{i j}+\right)$ e $\mu_{i j}\left(t_{i j}+\right)=\mu_{i j}(t)$ para $t>t_{i j}$.

Por exemplo, $\mu$ pode ser uma função escada. Neste caso

$$
\Delta_{0}(z)=I-\sum_{j=1}^{\infty} e^{-z r_{j}} A_{j}
$$

O próximo lema é uma adaptação do Teorema 4.6 de Verduyn Lunel [10]. Recordamos que $\mathbb{C}_{\gamma_{1}, \gamma_{2}}=\left\{z \in \mathbb{C}: \gamma_{1}<\operatorname{Re} z<\gamma_{2}\right\}$.

Lema 3.1. Se $\mu$ satistaz (J), então os zeros de det $\Delta_{0}(z)$ estão localizados em uma faixa finita $\mathbb{C}_{\alpha_{0}, \omega_{0}}$. Para z na faixa $\mathbb{C}_{\omega_{0}, \infty}$, existem constantes positivas m, $M$ e $\tau$ tais que

$$
m \leqslant\left|\operatorname{det} \Delta_{0}(z)\right| \leqslant M
$$

Além disso, para qualquer $\epsilon>0$, para uma escolha adequada de $m$ e $M$, a estimativa (3.3) vale para $z \in \mathbb{C}_{\alpha_{0}, \omega_{0}}$ fora de círculos de raio $\epsilon$ centrados nos zeros de $\operatorname{det} \Delta_{0}(\cdot)$.

Demonstração. Dividiremos a prova em quatro partes. Na parte 1, escrevemos det $\Delta_{0}(z)$ como a transformada de Laplace-Stieltjes de uma medida $d \tilde{\mu}$. Na parte 2 , mostraremos que existe $\alpha_{0}$ tal que não há raízes de $\operatorname{det} \Delta_{0}(z)$ no semiplano $\mathbb{C}_{-\infty, \alpha_{0}}$. Na parte 3 , mostraremos o mesmo para $\mathbb{C}_{\omega_{0}, \infty}$ e a estimativa (3.3) nesta região. Finalmente, na parte 4 , mostraremos a estimativa (3.3) na faixa $\mathbb{C}_{\alpha_{0}, \omega_{0}}$.

Parte 1: Para qualquer função $\alpha \in N B V\left([0, r], \mathbb{C}^{n \times n}\right)$, temos que

$$
\int_{0}^{r} e^{-z \theta} d \alpha(\theta)=\int_{0}^{\infty} e^{-z \theta} d \alpha(\theta)=\mathcal{L}(d \alpha)(z)
$$

Temos que $\mathcal{L}\left(d h_{y}\right)(z)=e^{-z y}$, onde $h_{y}$ é a função de Heaviside. Podemos reescrever $\Delta_{0}(z)$, dado por $(3.2)$, como

$$
\Delta_{0}(z)=I-\int_{0}^{r} e^{-z \theta} d \mu(\theta)=e^{-0 z} I-\int_{0}^{\infty} e^{-z \theta} d \mu(\theta)
$$




$$
\begin{aligned}
& =\mathcal{L}\left(d h_{0} I\right)(z)-\mathcal{L}(d \mu)(z)=\mathcal{L}\left(d h_{0} I-d \mu\right)(z) \\
& =\mathcal{L}(d \xi)(z)
\end{aligned}
$$

onde $\xi_{i j}=\delta_{i j} h_{0}-\mu_{i j}$. Como det $\Delta_{0}(z)$ é uma soma de produtos de $\mathcal{L}\left(d \mu_{i j}\right)(z)$, que é igual à transformada de Laplace-Stieltjes da soma de certas convoluções de $d \mu_{i j}$, existe uma função $\tilde{\mu}$ de variação limitada tal que

$$
\operatorname{det} \Delta_{0}(z)=\int_{0}^{\tau} e^{-z s} d \tilde{\mu}(s)
$$

onde $\tilde{\mu}$ satisfaz $(\mathrm{J})$, tem necessariamente um salto em 0 e num último ponto $\tau \leqslant n r$.

Parte 2: Seja agora $l=|\tilde{\mu}(\tau)-\tilde{\mu}(\tau-)| / 2$ e escolha $\epsilon>0$ tal que $\operatorname{Var}_{[\tau-\epsilon, \tau)} \tilde{\mu}<\frac{l}{2}$. Sejam $\gamma=\min \left\{l /\left(2 \operatorname{Var}_{[0, \tau-\epsilon]} \tilde{\mu}\right), 1\right\}$ e $\alpha_{0}=\frac{1}{\epsilon} \ln \gamma \leqslant 0$. Então, para $z \in \mathbb{C}_{-\infty, \alpha_{0}}$, temos que

$$
\left|e^{z \epsilon}\right|=e^{\operatorname{Re} z \epsilon} \leqslant e^{\alpha_{0} \epsilon}=\gamma \leqslant \frac{l}{2 \operatorname{Var}_{[0, \tau-\epsilon]} \tilde{\mu}} .
$$

Portanto, para $z \in \mathbb{C}_{-\infty, \alpha_{0}}$,

$$
\begin{aligned}
\left|\int_{0}^{\tau} e^{-z t} d \tilde{\mu}(t)\right| & =\left|e^{-z \tau}(\tilde{\mu}(\tau)-\tilde{\mu}(\tau-))+\int_{0}^{\tau-\epsilon} e^{-z t} d \tilde{\mu}(t)+\int_{\tau-\epsilon}^{\tau-} e^{-z t} d \tilde{\mu}(t)\right| \\
& \geqslant\left|e^{-z \tau}\right|\left[|\tilde{\mu}(\tau)-\tilde{\mu}(\tau-)|-\left|e^{z \epsilon}\right| \operatorname{Var}_{[0, \tau-\epsilon]} \tilde{\mu}-\operatorname{Var}_{[\tau-\epsilon, \tau)} \tilde{\mu}\right] \\
& \geqslant\left|e^{-z \tau}\right|\left[2 l-\frac{l}{2}-\frac{l}{2}\right]=l\left|e^{-z \tau}\right| .
\end{aligned}
$$

Portanto, para $\alpha_{0}=\frac{1}{\epsilon} \ln \gamma \leqslant 0$, segue que não há zeros de $\operatorname{det} \Delta_{0}(z)=$ $\int_{0}^{\tau} e^{-z t} d \tilde{\mu}(t)$ em $\mathbb{C}_{-\infty, \alpha_{0}}$.

Parte 3: Mostramos agora que existe $\omega_{0}$ tal que não há raízes de $\operatorname{det} \Delta_{0}(z)$ no semiplano $\mathbb{C}_{\omega_{0}, \infty}$. Seja $m$ dado por $m \stackrel{\text { def }}{=}|\tilde{\mu}(0)-\tilde{\mu}(0-)| / 2$. Escolha $\delta>0$ tal que $\operatorname{Var}_{(0, \delta]} \tilde{\mu}<\frac{m}{2}$. Sejam $\nu=\max \left\{\left(2 \operatorname{Var}_{[\delta, \tau]} \tilde{\mu}\right) / m, 1\right\}$ e $\omega_{0}=\frac{1}{\delta} \ln \nu \geqslant 0$. Então, para $z \in \mathbb{C}_{\omega_{0}, \infty}$,

$$
\left|e^{-z \delta}\right|=e^{-\operatorname{Re} z \delta} \leqslant e^{-\omega_{0} \delta}=e^{-\ln \nu}=\frac{1}{\nu} \leqslant \frac{m}{2 \operatorname{Var}_{[\delta, \tau]} \tilde{\mu}} .
$$

Portanto, para $z \in \mathbb{C}_{\omega_{0}, \infty}$, temos que

$$
\left|\int_{0}^{\tau} e^{-z t} d \tilde{\mu}(t)\right|=\left|(\tilde{\mu}(0)-\tilde{\mu}(0-))+\int_{0+}^{\delta} e^{-z t} d \tilde{\mu}(t)+\int_{\delta}^{\tau} e^{-z t} d \tilde{\mu}(t)\right|
$$




$$
\begin{aligned}
& \geqslant|\tilde{\mu}(0)-\tilde{\mu}(0-)|-\operatorname{Var}_{(0, \delta]} \tilde{\mu}-\left|e^{-z \delta}\right| \operatorname{Var}_{[\delta, \tau]} \tilde{\mu} \\
& \geqslant 2 m-\frac{m}{2}-\frac{m}{2}=m .
\end{aligned}
$$

Concluimos que, para $\omega_{0}=\frac{1}{\delta} \ln \nu \geqslant 0$, não há zeros de $\operatorname{det} \Delta_{0}(z)$ em $\mathbb{C}_{\omega_{0}, \infty}$. Assim, fica mostrado que os zeros de $\operatorname{det} \Delta_{0}(\cdot)$ estão localizados em uma faixa finita $\mathbb{C}_{\alpha_{0}, \omega_{0}}$. Além disso, sendo $M \stackrel{\text { def }}{=} \operatorname{Var}_{[0, \tau]} \tilde{\mu}$, temos que $\left|\int_{0}^{\tau} e^{-z t} d \tilde{\mu}(t)\right| \leqslant$ $M$ e, como $\operatorname{Re} z>\omega_{0} \geqslant 0$, segue a estimativa (3.3) para $z \in \mathbb{C}_{\omega_{0}, \infty}$.

Parte 4: Provemos agora que, para cada $\epsilon>0$, existem $m$ e $M$ tais que vale (3.3),

$$
m \leqslant\left|\operatorname{det} \Delta_{0}(z)\right| \leqslant M
$$

para $z \in \mathbb{C}_{\alpha_{0}, \omega_{0}}$, fora de círculos de raio $\epsilon$ centrados nos zeros de $\operatorname{det} \Delta_{0}$. Iniciamos com o limitante inferior para $\left|\operatorname{det} \Delta_{0}(z)\right|$.

Denote os zeros de det $\Delta_{0}$ por $\lambda_{1}, \lambda_{2}, \ldots$ e suponha que tal constante $m$ não exista. Então existem $\epsilon>0$ e uma sequência $z_{1}, z_{2}, \ldots$ de pontos em $\mathbb{C}_{\alpha_{0}, \omega_{0}}$, mas fora dos discos $\left|z-\lambda_{j}\right| \leqslant \epsilon$, tais que

$$
\lim _{j \rightarrow \infty} \operatorname{det} \Delta_{0}\left(z_{j}\right)=0
$$

Seja $z_{j}=x_{j}+i y_{j}$. Como $\sup _{j}\left|x_{j}\right| \leqslant \max \left\{\left|\alpha_{0}\right|,\left|\omega_{0}\right|\right\}$, podemos supor, sem perda de generalidade, que

$$
\lim _{j \rightarrow \infty} x_{j}=\bar{x} .
$$

Defina agora uma sequência de funções inteiras dadas por

$$
F_{j}(z)=\operatorname{det} \Delta_{0}\left(z+i y_{j}\right)
$$

Daí, como $\left|e^{-z t}\right|$ é limitada em faixas verticais onde a parte real é limitada e $\tilde{\mu} \in N B V\left([0, \tau], \mathbb{C}^{n \times n}\right)$, segue que $\operatorname{det} \Delta_{0}$ é limitada na vizinhança

$$
U=\left\{z: \alpha_{0}-1<\operatorname{Re} z<\omega_{0}+1\right\}
$$

da faixa fechada $\alpha_{0} \leqslant \operatorname{Re} z \leqslant \omega_{0}$. Segue que a sequência $\left\{F_{j}\right\}$ é uniformemente limitada em $U$ e, portanto, localmente limitada. Pelo Teorema de Montel $^{1},\left\{F_{j}\right\}$ forma uma família normal ${ }^{2}$. Ver, por exemplo, Conway [2].

\footnotetext{
${ }^{1}$ Seja $H(U) \subset \mathcal{C}(U, \mathbb{C})$ o conjunto das funções analíticas num aberto $U$; então uma família $\mathcal{H}$ em $H(U)$ é normal se, e somente se, $\mathcal{H}$ é localmente limitada.

${ }^{2}$ Seja $U \subset \mathbb{C}$ um aberto e $\left\{K_{n}\right\}$ uma sequência encaixante de compactos tais que
} 
Deste modo, existe uma subsequência $\left\{F_{j_{k}}\right\}$ que converge uniformemente em subconjuntos compactos da faixa $U$ para uma função limite $\bar{F}$. Além disso, $\bar{F} \not \equiv$ 0. Pelo Teorema VII.2.1 de Conway [2], sendo $\bar{F}$ limite de funções analíticas, segue que $\bar{F}$ é analítica. Como $F_{j}\left(x_{j}\right)=\operatorname{det} \Delta_{0}\left(z_{j}\right) \rightarrow 0$ quando $j \rightarrow \infty$, segue que $\bar{F}(\bar{x})=0$.

Mostremos agora que existe $\epsilon>0$ tal que $\bar{F}(z) \neq 0$ para $|z-\bar{x}|=\frac{\epsilon}{2}$. Para isto, seja $\delta$ tal que $\bar{B}(\bar{x}, \delta) \subset U$. Como os zeros de $\bar{F}$ são isolados, existe um número finito deles em $\bar{B}(\bar{x}, \delta)$ que denotaremos por $\bar{x}, \omega_{1}, \ldots, \omega_{n}$. Seja $\epsilon=\min \left\{\left|\omega_{j}-\bar{x}\right|\right\}>0$. Segue que $\bar{F}(z) \neq 0$ para $z$ tal que $|z-\bar{x}|=\frac{\epsilon}{2}$.

Como $\bar{F} \not \equiv 0$, pelo Teorema de Hurwitz ${ }^{3}$, temos que existe $N \in \mathbb{N}$ tal que, para $j \geqslant N, \bar{F}$ e $F_{j}$ tem o mesmo número de zeros em $B\left(\bar{x}, \frac{\epsilon}{2}\right)$, ou seja, um único zero. Podemos tomar tal $N$ suficientemente grande de modo que $\left|\bar{x}-x_{N}\right| \leqslant \frac{\epsilon}{2}$. Logo, $F_{j}$ tem um zero em $B\left(\bar{x}, \frac{\epsilon}{2}\right)$ para $j \geqslant N$. Para $j=N$, seja $a$ tal que $|a-\bar{x}|<\frac{\epsilon}{2}$ e $F_{N}(a)=0$ e tome $\bar{z}=a+i y_{N}$. Então $\operatorname{det} \Delta_{0}(\bar{z})=0$. Assim,

$\left|\bar{z}-z_{N}\right|=\left|a+i y_{N}-\left(x_{N}+i y_{N}\right)\right|=\left|a-x_{N}\right| \leqslant|a-\bar{x}|+\left|\bar{x}-x_{N}\right|<\frac{\epsilon}{2}+\frac{\epsilon}{2}=\epsilon$,

o que contradiz a suposição de que $z_{j}$ estavam fora de círculos de raio $\epsilon$ centrados nos zeros de det $\Delta_{0}$. Portanto existe $m$ tal que $\left|\operatorname{det} \Delta_{0}(z)\right| \geqslant m$. Além disso, como $\left|e^{-z t}\right|$ é uma função limitada para $z \in \mathbb{C}_{\alpha_{0}, \omega_{0}}, t \in[0, \tau] \mathrm{e}$ $\tilde{\mu}$ é de variação limitada, existe $M$ tal que

$$
\left|\operatorname{det} \Delta_{0}(z)\right|=\left|\int_{0}^{\tau} e^{-z t} d \tilde{\mu}(t)\right| \leqslant \max _{0 \leqslant t \leqslant \tau}\left|e^{-z t}\right| \operatorname{Var}_{[0, \tau]} \tilde{\mu} \leqslant M
$$

de onde segue a última afirmação do lema.

O seguinte exemplo, ilustra que o determinante de $\Delta_{0}$ é uma soma de produtos de $\mathcal{L}\left(d \mu_{i j}\right)(z)$ e que existe uma função $\tilde{\mu}$ de variação limitada, tal $\overline{U=\bigcup_{i=i}^{\infty} K_{n} \text {. Defina } \rho_{n}(f, g)=\sup }\left\{d(f(z), g(z)): z \in K_{n}, \forall f, g \in \mathcal{C}(U, \Omega)\right\}$, onde $\Omega$ é um espaço métrico completo, e considere a métrica $\rho$ em $\mathcal{C}(U, \Omega)$ dada por $\rho(f, g)=$ $\sum_{n=1}^{\infty} 2^{-n} \frac{\rho_{n}(f, g)}{1+\rho_{n}(f, g)}$. Uma família $\mathcal{H} \subset \mathcal{C}(U, \Omega)$ é normal se cada sequência em $\mathcal{H}$ tem uma subsequência que converge para uma função $f \in \mathcal{C}(U, \Omega)$. Para $U$ dado em (3.7), temos $U=\bigcup_{n=1}^{\infty} K_{n}$ onde $K_{n}=\left\{z \in \mathbb{C}: \alpha_{0}-1+\frac{1}{n} \leqslant \operatorname{Re} z \leqslant \omega_{0}+1-\frac{1}{n}\right.$ e $\left.-n \leqslant \operatorname{Im} z \leqslant n\right\}$.

${ }^{3}$ Seja $U \subset \mathbb{C}$ e suponha que a sequência $\left\{f_{n}\right\}$ em $H(U)$ converge para $f$. Se $f \not \equiv 0$, existe $a \in U$ e $R>0$ tal que $\bar{B}(a, R) \subset U$ e $f(z) \neq 0$ em $|z-a|=R$, então existe $N \in \mathbb{Z}$ tal que, para $n \geqslant N, f$ e $f_{n}$ têm o mesmo número de zeros em $B(a, R)$. 
que

$$
\operatorname{det} \Delta_{0}(z)=\int_{0}^{\tau} e^{-z s} d \tilde{\mu}(s)
$$

onde $\tilde{\mu}$ satisfaz $(J)$ e $\tau$ é o ponto onde ocorre o último salto de $\tilde{\mu}$ que tem, necessariamente, um salto em 0.

Exemplo 3.2. Seja $\mu \in N B V\left([0, r], \mathbb{C}^{2 \times 2}\right)$ definida por

$$
\mu(\theta)=\left(\begin{array}{cc}
a h_{t_{0}}(\theta) & b h_{t_{1}}(\theta) \\
c h_{t_{2}}(\theta) & d h_{t_{3}}(\theta)
\end{array}\right)
$$

onde $a, b, c, d \in \mathbb{R}, t_{j}>0$ para $j=0,1,2,3$ e $h_{t}$ é a função de Heaviside.

Deste modo, recordando que $\mathcal{L}\left(d h_{t}\right)(z)=e^{-z t}, t>0$,

$$
\begin{aligned}
\Delta_{0}(z) & =I-\int_{0}^{r} e^{-z \theta} d \mu(\theta)=\left(\begin{array}{ll}
1 & 0 \\
0 & 1
\end{array}\right)-\left(\begin{array}{cc}
a \mathcal{L}\left(d h_{t_{0}}\right)(z) & b \mathcal{L}\left(d h_{t_{1}}\right)(z) \\
c \mathcal{L}\left(d h_{t_{2}}\right)(z) & d \mathcal{L}\left(d h_{t_{4}}\right)(z)
\end{array}\right) \\
& =\left(\begin{array}{cc}
1-a e^{-z t_{0}} & -b e^{-z t_{1}} \\
-c e^{-z t_{2}} & 1-d e^{-z t_{3}}
\end{array}\right) .
\end{aligned}
$$

Então, temos que

$$
\operatorname{det} \Delta_{0}(z)=1-d e^{-z t_{3}}-a e^{-z t_{0}}+a d e^{-z\left(t_{0}+t_{3}\right)}-b c e^{-z\left(t_{1}+t_{2}\right)} .
$$

Suponha ainda, sem perda de generalidade, que $t_{0}<t_{3}$ e $t_{0}+t_{3}<t_{1}+t_{2}$. Considere $\tilde{\mu}(\theta)$ a função de variação limitada definida em $\mathbb{R}$ dada por

$$
\tilde{\mu}(\theta)= \begin{cases}0, & \theta<0, \\ 1, & 0 \leqslant \theta<t_{0}, \\ 1-a, & t_{0} \leqslant \theta<t_{3}, \\ 1-a-d, & t_{3} \leqslant \theta<t_{0}+t_{3}, \\ 1-a-d+a d, & t_{0}+t_{3} \leqslant \theta<t_{1}+t_{2}, \\ 1-a-d+a d-b c, & \theta \geqslant t_{1}+t_{2} .\end{cases}
$$

Então, concluimos que $\operatorname{det} \Delta_{0}(z)=\int_{0}^{\tau} e^{-z \theta} d \tilde{\mu}(\theta)$ onde $\tau=t_{1}+t_{2}$ é o ponto onde ocorre o último salto de $\tilde{\mu}$.

Teorema 3.3. Suponha que $\mu$ satisfaz $(J)$ e tome $\mathbb{C}_{\alpha_{0}, \omega_{0}}$ como no Lema 3.1. Para qualquer $\delta>0$, existe $k$ tal que para qualquer zero $\zeta_{0} \operatorname{de} \operatorname{det} \Delta_{0}(\cdot)$ com 
$\left|\zeta_{0}\right|>k$ existe um zero $\zeta$ de $\operatorname{det} \Delta(\cdot) \operatorname{com}\left|\zeta_{0}-\zeta\right|<\delta$. Além disso, existem constantes positivas $m$ e $M$ tais que

$$
m \leqslant\left|\operatorname{det}\left(\frac{1}{z} \Delta(z)\right)\right| \leqslant M
$$

para $z \in \mathbb{C}_{\alpha_{0}, \omega_{0}}$ com $|z|>k$ e fora de círculos de raio $\epsilon$ centrados nos zeros de $\operatorname{det} \Delta(\cdot)$.

Demonstração. Para $|z|>1, \operatorname{det} \Delta(z)=0$ se, e somente se, $\operatorname{det}\left(\frac{1}{z} \Delta(z)\right)=0$ $\mathrm{e}$

$$
\frac{1}{z} \Delta(z)=\Delta_{0}(z)-\frac{1}{z} \int_{0}^{\tau} e^{-z \theta} d \eta(\theta) .
$$

Do Lema 3.1 e para $\delta$ suficientemente pequeno, existe $m_{1}$ tal que

$$
\left|\operatorname{det} \Delta_{0}(z)\right| \geqslant m_{1}
$$

para $z$ fora de círculos de raio $\delta$ centrados nas raízes de det $\Delta_{0}(\cdot)$. Podemos estimar

$$
\frac{1}{z}\left\|\int_{0}^{\tau} e^{-z \theta} d \eta(\theta)\right\|<\frac{1}{z} \int_{0}^{\tau} d|\eta|(\theta)\left|e^{-z \theta}\right| \leqslant \frac{1}{z} \operatorname{Var}_{[0, \tau]} \eta \max \left\{e^{-\alpha_{0} \tau}, 1\right\} \stackrel{\text { def }}{=} \frac{C}{z}
$$

onde $d|\eta|$ denota a variação total da medida de $d \eta$. Portanto, uma vez que o determinante é uma função contínua, para $|z|$ suficientemente grande, digamos $|z|>k$,

$$
\left|\operatorname{det}\left(\frac{1}{z} \Delta(z)\right)-\operatorname{det} \Delta_{0}(z)\right| \leqslant \frac{m_{1}}{2} .
$$

Sejam $\lambda_{1}, \lambda_{2}, \ldots$ os zeros de $\operatorname{det} \Delta_{0}(\cdot)$. Como, para todo $i, \operatorname{det} \Delta_{0}(z)$ e $\operatorname{det}\left(\frac{1}{z} \Delta(z)\right)$ são funções analíticas em uma vizinhança $\Omega$ de $\bar{B}\left(\lambda_{i}, \delta\right)$ sem zeros ou pólos em $\gamma_{i}=\left\{z:\left|z-\lambda_{i}\right|=\delta\right\}$ e

$$
\left|\operatorname{det}\left(\frac{1}{z} \Delta(z)\right)-\operatorname{det} \Delta_{0}(z)\right| \leqslant \frac{m_{1}}{2}<m_{1}<\left|\operatorname{det} \Delta_{0}(z)\right|
$$

segue, pelo Teorema de Rouché ${ }^{4}$ que $\operatorname{det}\left(\frac{1}{z} \Delta(z)\right)$ e $\operatorname{det} \Delta_{0}(z)$ tem o mesmo número de zeros dentro dos círculos de raio $\delta$ centrados nas raízes de det $\Delta_{0}(z)$.

\footnotetext{
${ }^{4}$ Suponha que $\gamma$ é um caminho fechado em uma região $\Omega$, tal que $\operatorname{Ind}_{\gamma}(\alpha)=0$ ou 1 , para todo $\alpha \in \Omega-\{\gamma\}$ (onde $\{\gamma\}$ é o traço de $\gamma$ ), e tome $\Omega_{1}$ como o conjunto de todos os $\alpha$ com $\operatorname{Ind}_{\gamma}(\alpha)=1$. Para toda $f \in H(\Omega)$, seja $N_{f}$ o número de zeros de $f$ em $\Omega_{1}$, contados de acordo com sua multiplicidade. Se $g \in H(\Omega)$ e $|f(z)-g(z)|<|f(z)|$, para todo $z \in\{\gamma\}$, então $N_{f}=N_{g}$.
} 
Pelo Lema 3.1, existe $M_{1}$ tal que $\left|\operatorname{det} \Delta_{0}(z)\right| \leqslant M_{1}$, daí, tomando $M=$ $\frac{m_{1}}{2}+M_{1}$, temos

$$
\begin{aligned}
\left|\operatorname{det}\left(\frac{1}{z} \Delta(z)\right)\right| & \leqslant\left|\operatorname{det}\left(\frac{1}{z} \Delta(z)\right)-\operatorname{det} \Delta_{0}(z)\right|+\left|\operatorname{det} \Delta_{0}(z)\right| \\
& \leqslant \frac{m_{1}}{2}+M_{1}=M .
\end{aligned}
$$

Também, tomando $m=\frac{m_{1}}{2}$, temos

$$
\begin{aligned}
\left|\operatorname{det}\left(\frac{1}{z} \Delta(z)\right)\right| & \geqslant\left|\operatorname{det} \Delta_{0}(z)\right|-\left|\operatorname{det}\left(\frac{1}{z} \Delta(z)\right)-\operatorname{det} \Delta_{0}(z)\right| \\
& \geqslant m_{1}-\frac{m_{1}}{2}=\frac{m_{1}}{2}=m .
\end{aligned}
$$

Portanto $m \leqslant\left|\operatorname{det}\left(\frac{1}{z} \Delta(z)\right)\right| \leqslant M$.

Lema 3.4. Seja $\xi \in N B V([0, r], \mathbb{C})$. Então

1. Para todo $C>0$, vale

$$
|\mathcal{L}(d \xi)(z)|<\frac{|z|}{C} \operatorname{Var}_{[0, r]} \xi
$$

$$
\text { para } z \in \mathbb{C} \text { tal que }|z|>C\left|e^{-z r}\right| e|z|>C \text {. }
$$

2. Para $\alpha \in \mathbb{R}$, vale

$$
|\mathcal{L}(d \xi)(z)|<e^{|\alpha| r} \operatorname{Var}_{[0, r]} \xi
$$

para $z \in \mathbb{C}$ tal que $\operatorname{Re} z>\alpha$.

Demonstração. Parte 1: Para $0 \leqslant \theta \leqslant r$ temos

$$
\left|e^{-z \theta}\right|=e^{-\theta \operatorname{Re} z}<\frac{|z|}{C}
$$

pois se $\operatorname{Re} z \geqslant 0$, então $e^{-\theta \operatorname{Re} z} \leqslant 1<|z| / C$ e se $\operatorname{Re} z<0$ então $e^{-\theta \operatorname{Re} z}<$ $e^{-r \operatorname{Re} z}=\left|e^{-z r}\right|<|z| / C$. Portanto,

$$
|\mathcal{L}(d \xi)(z)|=\left|\int_{0}^{r} e^{-z \theta} d \xi(\theta)\right| \leqslant \max _{\theta \in[0, r]}\left|e^{-z \theta}\right| \operatorname{Var}_{[0, r]} \xi<\frac{|z|}{C} \operatorname{Var}_{[0, r]} \xi
$$

como queríamos.

Parte 2: Para $0 \leqslant \theta \leqslant r$ temos que

$$
-\operatorname{Re} z \theta \leqslant-\alpha \theta \leqslant|\alpha| \theta \leqslant|\alpha| r,
$$


de onde concluimos que

$$
\left|e^{-z \theta}\right| \leqslant e^{|\alpha| r}
$$

Portanto,

$$
|\mathcal{L}(d \xi)(z)|=\left|\int_{0}^{r} e^{-z \theta} d \xi(\theta)\right| \leqslant \max _{\theta \in[0, r]}\left|e^{-z \theta}\right| \operatorname{Var}_{[0, r]} \xi \leqslant e^{|\alpha| r} \operatorname{Var}_{[0, r]} \xi,
$$

concluindo a demonstração.

Lema 3.5. Para $\operatorname{det} \Delta(z)$, temos a seguinte representação

$$
\operatorname{det} \Delta(z)=\operatorname{det} \Delta_{0}(z) z^{n}+\sum_{m=0}^{n-1} \mathcal{L}\left(d \xi_{m}\right)(z) z^{m}
$$

onde $d \xi_{m}$ são certas convoluções entre $\mu_{i j}$ e $\eta_{i j}$ para alguns índices $i$ e $j$.

Demonstração. Primeiro, observamos que, se $A$ e $B$ são duas matrizes $n \times n$, então $\operatorname{det}(z A+w B)$ é um polinômio de ordem $n$ em $z \mathrm{e} w$. Tomando $w=0$, vemos que o coeficiente do termo $z^{n}$ é $\operatorname{det} A$. De (3.1) e (3.2), a matriz $\Delta(z)$, dada por

$$
\begin{aligned}
\Delta(z)=z\left(I-\int_{0}^{r} e^{-z \theta} d \mu(\theta)\right)- & \int_{0}^{r} e^{-z \theta} d \eta(\theta) \\
& =z I-z \int_{0}^{r} e^{-z \theta} d \mu(\theta)-\int_{0}^{r} e^{-z \theta} d \eta(\theta)
\end{aligned}
$$

tem como entradas a soma de $z$ ou 0 (dependendo se a entrada está na diagonal) com elementos da forma $z \mathcal{L}\left(d \mu_{i j}\right)(z)$ e $\mathcal{L}\left(d \eta_{i j}\right)(z)$, onde $\mu_{i j}$ e $\eta_{i j}$ são as entradas das funções matriciais $\mu$ e $\eta$. Portanto, o determinante de $\Delta(z)$ consiste de somas de produtos de tais elementos. Agrupando elementos com a mesma potência de $z$, segue que os coeficientes são somas de convoluções entre $\mu_{i j}$ e $\eta_{i j}$ para alguns índices $i$ e $j$. Concluimos que o determinante de $\Delta(z)$ pode ser representado por (3.11), tomando $\xi_{k}$ como as somas das convoluções entre $\mu_{i j}$ e $\eta_{i j}$, que é coeficiente da potência $z^{k}$.

Exemplo 3.6. Sejam $\mu, \eta \in N B V\left([0,2], \mathbb{C}^{2 \times 2}\right)$ dadas por

$$
\mu(\theta)=\left(\begin{array}{cc}
h_{1}(\theta) & 2 h_{2}(\theta) \\
0 & h_{2}(\theta)
\end{array}\right) \quad \eta(\theta)=\left(\begin{array}{cc}
2 h_{0}(\theta) & h_{2}(\theta) \\
2 h_{1}(\theta) & 0
\end{array}\right)
$$


Temos que

$$
\begin{gathered}
\Delta_{0}(z)=I-\int_{0}^{r} e^{-z \theta} d \mu(\theta)=\left(\begin{array}{cc}
1-e^{-z} & -2 e^{-2 z} \\
0 & 1-e^{-2 z}
\end{array}\right), \\
\Delta(z)=z \Delta_{0}(z)-\int_{0}^{r} e^{-z \theta} d \eta(\theta)=\left(\begin{array}{cc}
z-z e^{-z}-2 & -2 z e^{-2 z}-e^{-2 z} \\
-2 e^{-z} & z-z e^{-2 z}
\end{array}\right),
\end{gathered}
$$

de onde segue que

$$
\operatorname{det} \Delta_{0}(z)=1-e^{-z}-e^{-2 z}+e^{-3 z} \text {, }
$$

$$
\operatorname{det} \Delta(z)=z^{2}\left(1-e^{-z}-e^{-2 z}+e^{-3 z}\right)+z\left(-2+2 e^{-2 z}-4 e^{-3 z}\right)-2 e^{-3 z} \text {. }
$$

Então, tomando $\xi_{0}$ e $\xi_{1}$ como

$$
\xi_{0}(\theta)=\left\{\begin{array}{ll}
0, & \theta<3, \\
-2, & \theta \geqslant 3,
\end{array} \quad \xi_{1}(\theta)= \begin{cases}0, & \theta<0 \text { ou } 2 \leqslant \theta<3 \\
-2, & 0 \leqslant \theta<2, \\
-4, & \theta \geqslant 3,\end{cases}\right.
$$

temos que (3.12) é escrita na forma (3.11) com $n=2$.

Definição 3.7. Seja $a_{M}$ definido por

$$
a_{M}=\inf \left\{\lambda \in \mathbb{R}: \#_{\Delta_{0}}(\lambda)<\infty\right\}
$$

onde $\#_{\Delta_{0}}(\lambda)$ é o número de zeros de $\operatorname{det} \Delta_{0}(z)$ em $\mathbb{C}_{\lambda, \infty}$, isto é, para cada $\epsilon>0$, existe um número finito de zeros $\zeta \operatorname{de} \operatorname{det} \Delta_{0}(z)$ tais que $\operatorname{Re} \zeta>a_{M}+\epsilon$.

Observação 3.8. Aplicando o Teorema 3.3, podemos substituir $\#_{\Delta_{0}}$ por $\#_{\Delta}$ em (3.13), onde $\#_{\Delta}(\lambda)$ é definido como o número de zeros do $\operatorname{det} \Delta(z) \mathrm{em}$ $\mathbb{C}_{\lambda, \infty}$.

Teorema 3.9. Para qualquer $\epsilon>0$, existem constantes positivas $q, C_{0}$ e $C$ tais que

$$
|\operatorname{det} \Delta(z)| \geqslant q|z|^{n}
$$

para todo $z \in \mathbb{C}$ tal que $|z|>C_{0}\left|e^{-z r}\right|,|z|>C$ e $\operatorname{Re} z>a_{M}+\epsilon$. 
Demonstração. Do Lema 3.1, existe $q$ tal que

$$
\left|\operatorname{det} \Delta_{0}(z)\right| \geqslant 2 q
$$

para $z$ satisfazendo Re $z>a_{M}+\epsilon$ e $z$ fora de círculos de raio 1 centrados nos zeros de det $\Delta_{0}(\cdot)$. Pela Definição 3.7 e Observação 3.8, temos que existe um número finito de zeros de $\operatorname{det} \Delta(z)$ e $\operatorname{det} \Delta_{0}(z) \operatorname{com} \operatorname{Re} z>a_{M}+\epsilon$. Seja

$$
C_{0}=\max \left\{|z|: \operatorname{Re} z>a_{M}+\epsilon \mathrm{e} \operatorname{det} \Delta(z) \operatorname{det} \Delta_{0}(z)=0\right\}+1,
$$

Então para $z \in \mathbb{C}$ tal que $\operatorname{Re} z>a_{M}+\epsilon$ e $|z|>C_{0}$, vale (3.15). Do Lema 3.5, deduzimos que

$$
|\operatorname{det} \Delta(z)| \geqslant\left.|| \operatorname{det} \Delta_{0}(z)|| z\right|^{n}-\sum_{m=0}^{n-1}\left|\mathcal{L}\left(d \xi_{m}\right)(z)\right||z|^{m} \mid .
$$

Aplicando o Lema 3.4 para cada $\xi_{m}$, temos que para todo $C$ e para todo $z \in \mathbb{C}$ tal que $|z|>C\left|e^{-z r}\right|$ e $|z|>C$,

$$
\begin{aligned}
\sum_{m=0}^{n-1}\left|\mathcal{L}\left(d \xi_{m}\right)(z)\right||z|^{m} & \leqslant \sum_{m=0}^{n-1} \frac{\operatorname{Var} \xi_{m}}{C}|z|^{m+1}=\sum_{m=0}^{n-1} \frac{\operatorname{Var} \xi_{m}}{C}|z|^{m+1-n}|z|^{n} \\
& =|z|^{n} \sum_{m=0}^{n-1} \frac{\operatorname{Var} \xi_{m}}{C}|z|^{m+1-n}<|z|^{n} \sum_{m=0}^{n-1} \operatorname{Var} \xi_{m} C^{m-n}
\end{aligned}
$$

uma vez que $m-n+1 \leqslant 0$ para $m \leqslant n-1$. Então, tomando $\varrho(C)=$ $\sum_{m=0}^{n-1} \operatorname{Var} \xi_{m} C^{m-n}$ temos que

$$
|\operatorname{det} \Delta(z)| \geqslant|z|^{n}\left(\left|\operatorname{det} \Delta_{0}(z)\right|-\varrho(C)\right)>|z|^{n}(2 q-\varrho(C))
$$

e então, escolhendo $C>C_{0}$ suficientemente grande tal que $\varrho(C)<q$ temos

$$
|\operatorname{det} \Delta(z)|>|z|^{n}\left(2 q-\varrho\left(C_{0}, C\right)\right)>q|z|^{n} \text {. }
$$

O próximo Lema e seu Corolário serão úteis no cálculo do contorno de integrais.

Lema 3.10. Suponha que $\mu$ satisfaz $(J)$. Para todo $f \in \mathcal{F}$,

$$
\lim _{\operatorname{Im} z \rightarrow \pm \infty} e^{z t} \Delta(z)^{-1}\left(f(0)+\int_{0}^{r} e^{-z \theta} d f(\theta)\right)=0
$$

uniformemente para $z \in \mathbb{C}_{\gamma_{1}, \gamma_{2}}$ com $a_{M}<\gamma_{1}<\gamma_{2}$ e $t$ em conjuntos compactos. 
Demonstração. Tome $\epsilon=\frac{\left(\gamma_{1}-a_{M}\right)}{2}$. Pelo Teorema 3.9, existe uma constante positiva $q$ tal que, para $|z|$ suficientemente grande, temos

$$
|\operatorname{det} \Delta(z)| \geqslant q|z|^{n}
$$

Seja $\operatorname{adj} \Delta(z)$ a matriz adjunta de $\Delta(z)$. Recordamos que adj $\Delta(z)$ é a transposta da matriz dos cofatores cof $\Delta(z)$ de $\Delta(z)$, isto é, cada entrada de $\operatorname{adj} \Delta(z)$ é o determinante de uma submatriz $(n-1) \times(n-1)$ de $\Delta(z)$. Podemos aplicar para cada entrada de $\operatorname{adj} \Delta(z)$ os Lemas 3.1, 3.4 e 3.5, obtendo que cada entrada é da forma

$$
(\operatorname{adj} \Delta(z))_{i j}=\operatorname{cof} \Delta_{0}(z)_{j i} z^{n-1}+\sum_{k=0}^{n-2} \mathcal{L}\left(d \xi_{k_{i j}}\right)(z) z^{m}
$$

onde para $z \in \mathbb{C}_{\gamma_{1}, \gamma_{2}}$ e $|z|$ suficientemente grande

$$
m_{i j} \leqslant\left|\operatorname{cof} \Delta_{0}(z)_{j i}\right| \leqslant M_{i j}
$$

e

$$
\left|\mathcal{L}\left(d \xi_{k_{i j}}\right)(z)\right|<\operatorname{Var}_{[0, r]} \xi_{k_{i j}} e^{\left|\gamma_{1}\right| r} .
$$

Das desigualdades (3.19) e (3.20) temos que $\operatorname{det}\left(\Delta_{0}(z)\right)_{i j}$ é limitado inferiormente e $\mathcal{L}\left(d \xi_{k_{i j}}\right)(z)$ é limitada superiormente. Portanto, para $z \in \mathbb{C}_{\gamma_{1}, \gamma_{2}}$ com $|z|$ suficientemente grande, temos que existe $K_{0}>0$ tal que

$$
\left|(\operatorname{adj} \Delta(z))_{i j}\right| \leqslant K_{0}|z|^{n-1}
$$

Como

$$
\Delta(z)^{-1}=\frac{1}{\operatorname{det} \Delta(z)} \operatorname{adj} \Delta(z)
$$

obtemos que

$$
\left|\left(\Delta(z)^{-1}\right)_{i j}\right| \leqslant \frac{K_{0}}{q|z|} .
$$

Como $e^{z t}$ e $\int_{0}^{r} e^{-z \theta} d f(\theta)$ são uniformemente limitados para $z \in \mathbb{C}_{\gamma_{1}, \gamma_{2}}$ e $t$ em intervalos compactos, existe uma constante $K_{1}$ tal que

$$
\left|e^{z t} \Delta(z)^{-1}\left(f(0)+\int_{0}^{r} e^{-z t} d f(t)\right)\right| \leqslant \frac{K_{1}}{|z|} .
$$

Quando $\operatorname{Im} z \rightarrow \pm \infty$, temos que $|z| \rightarrow \infty$ e segue (3.17). 
Corolário 3.11. Seja $\beth_{N\left(\gamma_{1}, \gamma_{2}\right)}, N \in \mathbb{R}$ e $a_{M}<\gamma_{1}<\gamma_{2}$, o segmento de reta horizontal ligando $\gamma_{1}+i N$ a $\gamma_{2}+i N$. Então, para cada $t>0$,

$$
\lim _{N \rightarrow \pm \infty} \int_{\beth_{N\left(\gamma_{1}, \gamma_{2}\right)}} e^{z t} \Delta(z)^{-1}\left(f(0)+\int_{0}^{r} e^{-z \theta} d f(\theta)\right) d z=0 .
$$

\subsection{Comportamento assintótico para $t \rightarrow \infty$}

Nesta seção, obtemos, a partir dos resultados da seção 3.1 e da solução da equação de renovação dada no Teorema 2.17, os ingredientes necessários para estudar o comportamento assintótico das soluções da equação de renovação

$$
x=d k * x+f
$$

onde $k(\theta)=\mu(\theta)+\int_{0}^{\theta} \eta(s) d s$ e $\mu$ satisfaz a condição $(\mathrm{J})$ na página 34 .

Mostremos que, pela fórmula de inversão (1.12), na página 9, segue que o valor da integral complexa em (2.49), na página 31, independe da escolha de $\gamma>\gamma_{0}$ para algum $\gamma_{0}$ suficientemente grande. Denotaremos

$$
Q(z)=\Delta(z)^{-1}\left(f(0)+\int_{0}^{r} e^{-z \theta} d f(\theta)\right)
$$

e definiremos $\Gamma$ como o contorno fechado no plano complexo orientado positivamente que consiste de quatro segmentos de retas passando pelos vértices $\gamma_{1}-i N, \gamma_{1}+i N, \gamma+i N, \gamma-i N$. Tomando $\gamma_{0}$ como o ínfimo dos $\gamma$ tais que o semi-plano $\operatorname{Re} z>\gamma$ é livre de raízes de det $\Delta(z)$ e sabendo que $Q$ é analítica no semi-plano $\operatorname{Re} z>\gamma_{0}$, o Teorema de Cauchy nos diz que

$$
\frac{1}{2 \pi i} \int_{\Gamma} e^{z t} Q(z) d z=0
$$

para $\gamma_{0}<\gamma_{1}<\gamma$. Tomando o limite quando $N \rightarrow \infty$ e usando o limite no Corolário 3.11, temos

$$
\lim _{N \rightarrow \pm \infty} \int_{\gamma_{1}+i N}^{\gamma+i N} e^{z t} Q(z) d z=0
$$

e então, concluimos que

$$
\frac{1}{2 \pi i} \int_{\left(\gamma_{1}\right)} e^{z t} Q(z) d z=\frac{1}{2 \pi i} \int_{(\gamma)} e^{z t} Q(z) d z
$$


Entretanto, a estimativa (3.24) ainda é válida para $a_{M}<\gamma_{1}<\gamma$ e, obviamente $\gamma_{0} \geqslant a_{M}$. Assim, podemos mover a linha vertical $\operatorname{Re} z=\gamma_{1}$ da integração em (3.25) para $a_{M}<\gamma_{1}<\gamma_{0}$ tal que não há zeros com parte real $\gamma_{1}$. Neste caso, o resultado da integral de linha (3.23), de acordo com o Teorema de Cauchy, torna-se

$$
\frac{1}{2 \pi i} \int_{\Gamma} e^{z t} Q(z) d z=\sum_{j=1}^{m} \operatorname{Res}_{z=\lambda_{j}} e^{z t} Q(z)
$$

onde $\lambda_{j}, 1 \leqslant j \leqslant m$ são todas as raízes $\operatorname{de} \operatorname{det} \Delta(z)$ tais que $\operatorname{Re} z>\gamma_{1}$. Portanto,

$$
\frac{1}{2 \pi i} \int_{(\gamma)} e^{z t} Q(z) d z=\sum_{j=1}^{m} \operatorname{Res}_{z=\lambda_{j}} e^{z t} Q(z)+\frac{1}{2 \pi i} \int_{\left(\gamma_{1}\right)} e^{z t} Q(z) d z
$$

para $\gamma>\gamma_{0}$. A partir destas considerações, conseguimos os próximos resultados. O principal deles, o Teorema 3.17, relaciona o comportamento das soluções da equação (2.48). Começamos com o Lema de Riemann-Lebesgue.

Lema 3.12. Se $f \in L^{1}(\mathbb{R})$, então

$$
\lim _{t \rightarrow \pm \infty}\left|\int_{-\infty}^{\infty} e^{i t \omega} f(\omega) d \omega\right|=0
$$

Demonstração. Primeiro mostremos que, se $\lambda$ é a medida de Lebesgue usual e $f$ é qualquer função em $L^{1}(\mathbb{R})$, então

$$
\lim _{h \rightarrow 0}\left\|f_{h}-f\right\|_{1}=0
$$

onde $f_{h}(\omega) \stackrel{\text { def }}{=} f(\omega+h)$. Seja $\epsilon>0$ arbitrário e considere $\mathcal{C}_{00}(\mathbb{R})=\left\{\varphi \in \mathcal{C}(\mathbb{R}):\right.$ tal que existe $F$ é compacto, $\left.\varphi(x)=0, \forall x \in F^{c} \cap \mathbb{R}\right\}$ De Hewitt \& Stromberg [6], temos que $\mathbb{C}_{00}(\mathbb{R})$ é denso em $L^{1}(\mathbb{R})$, logo podemos escolher $\varphi \in \mathcal{C}_{00}(\mathbb{R})$ tal que $\|\varphi-f\|_{1}<\frac{\epsilon}{3}$. Como $\mathbb{R}$ é um espaço métrico localmente compacto, segue que todas as funções em $\mathcal{C}_{00}(\mathbb{R})$ são uniformemente contínuas. Portanto, $\varphi$ é uniformemente contínua e existe $\alpha \in \mathbb{R}, \alpha>0$ tal que $\varphi(t)=0$ se $|t| \geqslant \alpha$. Escolha $0<\delta<1$ tal que

$$
|\varphi(t+h)-\varphi(t)|<\frac{\epsilon}{3}\left(\frac{1}{2 \alpha}\right) \quad \text { para } \quad|h|<\delta
$$


Então, $|h|<\delta$ implica que $\int_{-\infty}^{\infty}|\varphi(t+h)-\varphi(t)| d t=\int_{-\alpha}^{\alpha}|\varphi(t+h)-\varphi(t)| d t<$ $\frac{\epsilon}{3}$, que é o mesmo que $\left\|\varphi_{h}-\varphi\right\|_{1}<\frac{\epsilon}{3}$. Também temos que $\left\|\varphi_{h}-f_{h}\right\|_{1}=$ $\|\varphi-f\|_{1}$ para todo $h>0$. Portanto, $\left\|f_{h}-f\right\|_{1} \leqslant\left\|f_{h}-\varphi_{h}\right\|_{1}+\left\|\varphi_{h}-\varphi\right\|_{1}+$ $\|\varphi-f\|_{1}<\epsilon$, se $|h|<\delta$ e então, $\lim _{h \rightarrow 0}\left\|f_{h}-f\right\|_{1}=0$.

Considerando $\hat{f}$ definido por

$$
\begin{aligned}
\hat{f}(t) & =\int_{-\infty}^{\infty} e^{i t \omega} f(\omega) d \omega=(-1) e^{-\pi i} \int_{-\infty}^{\infty} e^{i t \omega} f(\omega) d \omega \\
& =(-1) \int_{-\infty}^{\infty} e^{i\left(\omega-\frac{\pi}{t}\right) t} f(\omega) d \omega=(-1) \int_{-\infty}^{\infty} f\left(\omega+\frac{\pi}{t}\right) e^{i t \omega} d \omega
\end{aligned}
$$

temos que

$$
\begin{aligned}
2|\hat{f}(t)| & =\left|\int_{-\infty}^{\infty} e^{i t \omega} f(t) d \omega-\int_{-\infty}^{\infty} e^{i t \omega} f\left(\omega+\frac{\pi}{t}\right) d \omega\right| \\
& \leqslant \int_{-\infty}^{\infty}\left|f\left(\omega+\frac{\pi}{t}\right)-f(\omega)\right|\left|e^{i t \omega}\right| d \omega \\
& =\int_{-\infty}^{\infty}\left|f\left(\omega+\frac{\pi}{t}\right)-f(\omega)\right| d \omega=\left\|f_{\frac{\pi}{t}}-f\right\|_{1}
\end{aligned}
$$

de onde segue que $|\hat{f}(t)|$ é arbitrariamente pequeno se $|t|$ é suficientemente grande.

Observação 3.13. Uma aplicação do lema anterior nos dá o mesmo resultado para $f \in L^{1}\left(R_{+}\right)$, ou seja,

$$
\lim _{t \rightarrow \pm \infty}\left|\int_{0}^{\infty} e^{i t \omega} f(\omega) d \omega\right|=0 .
$$

Lema 3.14. Para $t>0$,

$$
\lim _{N \rightarrow \infty} \int_{-N}^{N} \frac{e^{i \omega t}}{\gamma+i \omega} d \omega= \begin{cases}2 \pi e^{-\gamma t}, & \gamma>0 \\ \pi, & \gamma=0 \\ 0, & \gamma<0 .\end{cases}
$$

Demonstração. De fato, temos que

$$
\frac{e^{i \omega t}}{\gamma+i \omega}=\frac{\gamma \cos (\omega t)}{\gamma^{2}+\omega^{2}}+\frac{\omega \operatorname{sen}(\omega t)}{\gamma^{2}+\omega^{2}}+i\left[\frac{\gamma \operatorname{sen}(\omega t)}{\gamma^{2}+\omega^{2}}-\frac{\omega \cos (\omega t)}{\gamma^{2}+\omega^{2}}\right]
$$

e, para todo $N \in \mathbb{R}$ temos que

$$
\int_{-N}^{N} \frac{\gamma \operatorname{sen}(\omega t)}{\gamma^{2}+\omega^{2}} d \omega=0 \quad \text { e } \quad \int_{-N}^{N} \frac{\omega \cos (\omega t)}{\gamma^{2}+\omega^{2}} d \omega=0
$$


uma vez que $\omega \mapsto \frac{\gamma \operatorname{sen}(\omega t)}{\gamma^{2}+\omega^{2}}$ e $\omega \mapsto \frac{\omega \cos (\omega t)}{\gamma^{2}+\omega^{2}}$ são funções ímpares. Além disso, também temos que

$$
\begin{aligned}
& \int_{-N}^{N} \frac{\gamma \cos (\omega t)}{\gamma^{2}+\omega^{2}} d \omega=2 \int_{0}^{N} \frac{\gamma \cos (\omega t)}{\gamma^{2}+\omega^{2}} d \omega \\
& \int_{-N}^{N} \frac{\omega \operatorname{sen}(\omega t)}{\gamma^{2}+\omega^{2}} d \omega=2 \int_{0}^{N} \frac{\omega \operatorname{sen}(\omega t)}{\gamma^{2}+\omega^{2}} d \omega,
\end{aligned}
$$

já que $\omega \mapsto \frac{\gamma \cos (\omega t)}{\gamma^{2}+\omega^{2}}$ e $\omega \mapsto \frac{\omega \operatorname{sen}(\omega t)}{\gamma^{2}+\omega^{2}}$ são funções pares. Logo, temos que

$$
\lim _{N \rightarrow \infty} \int_{-N}^{N} \frac{e^{i \omega t}}{\gamma+i \omega} d \omega=\lim _{N \rightarrow \infty} 2\left[\int_{0}^{N} \frac{\gamma \cos (\omega t)}{\gamma^{2}+\omega^{2}} d \omega+\int_{0}^{N} \frac{\omega \operatorname{sen}(\omega t)}{\gamma^{2}+\omega^{2}} d \omega\right] .
$$

Sabemos que (cf. Conway [2, p. 121]), se $a \geqslant 0$, então

$$
\int_{0}^{\infty} \frac{\cos (a x)}{1+x^{2}} d x=\frac{\pi}{2} e^{-a} \quad \text { e } \quad \int_{0}^{\infty} \frac{\cos (a t)}{\left(1+x^{2}\right)^{2}} d x=\frac{\pi(a+1)}{4} e^{-a} .
$$

Para resolver as integrais em (3.27), consideraremos três casos distintos: quando $\gamma>0, \gamma<0$ e $\gamma=0$.

Primeiramente, considere $\gamma>0$. Escrevendo $\omega t=\gamma t \frac{\omega}{\gamma}, \gamma^{2}+\omega^{2}=$ $\gamma^{2}\left(1+\frac{\omega^{2}}{\gamma^{2}}\right)$ e considerando a mudança de variável $x=\frac{\omega}{\gamma}$ e fazendo uma integração por partes, temos que o segundo membro de (3.27) torna-se

$$
\begin{array}{r}
2\left[\int_{0}^{\infty} \frac{\cos ((\gamma t) x)}{1+x^{2}} d x+\frac{2}{\gamma t} \int_{0}^{\infty} \frac{\cos ((\gamma t) x)}{\left(1+x^{2}\right)^{2}} d x-\frac{1}{\gamma t} \int_{0}^{\infty} \frac{\cos ((\gamma t) x)}{1+x^{2}} d x\right] \\
=2 \pi e^{-\gamma t}
\end{array}
$$

Para o caso, $\gamma<0$, da mesma forma como antes, o segundo membro de (3.27) torna-se

$$
\begin{array}{r}
2\left[-\int_{0}^{\infty} \frac{\cos ((-\gamma t) x)}{1+x^{2}} d x-\frac{2}{\gamma t} \int_{0}^{\infty} \frac{\cos ((-\gamma t) x)}{\left(1+x^{2}\right)^{2}} d x+\frac{1}{\gamma t} \int_{0}^{\infty} \frac{\cos (-\gamma t) x)}{1+x^{2}} d x\right] \\
=0 .
\end{array}
$$

Agora, se $\gamma=0$, então de (3.27) temos

$$
\lim _{N \rightarrow \infty} \int_{-N}^{N} \frac{e^{i \omega t}}{i \omega} d \omega=\lim _{N \rightarrow \infty} 2 \int_{0}^{N} \frac{\operatorname{sen}(\omega t)}{\omega}=\pi
$$


Lema 3.15. Para qualquer $\gamma>a_{M}$ tal que $\gamma \neq 0$ e não há zeros de $\operatorname{det} \Delta(z)$ $e \operatorname{det} \Delta_{0}(z)$ com $\operatorname{Re} z=\gamma$, temos que

$$
\frac{1}{2 \pi i} \int_{(\gamma)} e^{z t} \Delta(z)^{-1}\left(f(0)+\int_{0}^{r} e^{-z \theta} d f(\theta)\right) d z=o\left(e^{\gamma t}\right) \text { para } t \rightarrow \infty .
$$

Demonstração. Seja $Q(z)$ como em (3.22), na página 45. Devemos mostrar que

$$
\lim _{t \rightarrow \infty}\left(\lim _{N \rightarrow \infty} \int_{-N}^{N} e^{i t \omega} Q(\gamma+i \omega) d \omega\right)=0 .
$$

Na demonstração do Lema 3.10, vimos em (3.21) que, para $|\omega|$ suficientemente grande, existe $K_{1}$ tal que

$$
|Q(\gamma+i \omega)| \leqslant \frac{K_{1}}{|\gamma+i \omega|}
$$

mas isto não garante que $Q(\gamma+i \omega)$ é uma função $L^{1}$ em $\omega$, ou seja, a integral acima não necessariamente converge absolutamente e, então, não podemos aplicar o Lema de Riemann-Lebesgue 3.12. Porém, para qualquer $N$ fixo, o Lema de Riemann-Lebesgue nos diz que

$$
\lim _{t \rightarrow \infty} \int_{-N}^{N} e^{i t \omega} Q(\gamma+i \omega) d \omega=0
$$

Então, se provarmos que os limites $t \rightarrow \infty$ e $N \rightarrow \infty$ podem ser trocados, pelo menos, para alguns termos da integral (3.31), obtemos a conclusão desejada para esses termos. Portanto, é suficiente mostrar que a convergência para $N \rightarrow \infty$ é uniforme para $t \geqslant t_{0}$ para algum $t_{0}$ fixo.

Para $\xi \in N B V([0, r], \mathbb{C})$, a integral $\int_{0}^{r} e^{-z \theta} d \xi(\theta)$ é uniformemente limitada para $z=\gamma+i \omega \operatorname{com} \omega \in \mathbb{R}$. Como, por (3.1),

$$
\Delta(z)=z \Delta_{0}(z)-\int_{0}^{r} e^{-z \theta} d \eta(\theta)
$$

segue que, para $z=\gamma+i \omega$,

$$
\begin{array}{cc}
\operatorname{det} \Delta(z)=z^{n} \operatorname{det} \Delta_{0}(z)+\mathrm{O}\left(|\omega|^{n-1}\right), & |\omega| \rightarrow \infty, \\
\operatorname{adj} \Delta(z)=z^{n-1} \operatorname{adj} \Delta_{0}(z)+\mathrm{O}\left(|\omega|^{n-2}\right), & |\omega| \rightarrow \infty .
\end{array}
$$

Como $\Delta(z)^{-1}=\frac{1}{\operatorname{det} \Delta(z)}$ adj $\Delta(z)$, após alguns cálculos, concluimos que

$$
\Delta(z)^{-1}=\frac{1}{z} \Delta_{0}(z)^{-1}+O\left(|\omega|^{-2}\right) .
$$


Então, de (3.22) temos que

$$
Q(z)=\frac{1}{z} \Delta_{0}(z)^{-1}\left(f(0)+\int_{0}^{r} e^{-z \theta} d f(\theta)\right)+\mathrm{O}\left(|\omega|^{-2}\right) .
$$

O termo $\mathrm{O}\left(|\omega|^{-2}\right)$ é integrável em $\mathbb{R}$ e do Lema de Riemann-Lebesgue 3.12 segue que

$$
\lim _{t \rightarrow \infty} \int_{-\infty}^{\infty} e^{i t \omega} \mathrm{O}\left(|\omega|^{-2}\right) d \omega=0 .
$$

Portanto, podemos nos concentrar no primeiro termo. Um aplicação do Lema 3.1 nos garante que, para $\gamma>a_{M}$ tal que não há zeros de $\operatorname{det} \Delta_{0}(z)$ com $\operatorname{Re} z=\gamma$, existe um $\epsilon_{\gamma}>0$ tal que $\left|\operatorname{det} \Delta_{0}(z)\right|>\epsilon_{\gamma}$ para $z \in \mathbb{C}$ tal que $\operatorname{Re} z \geqslant \gamma$.

Afirmamos que $\Delta_{0}(z)^{-1}$ é a transformada de Laplace-Stieltjes de alguma função $N B V$, isto é, existe um $\zeta \in N B V\left([0, r], \mathbb{C}^{n \times n}\right)$ tal que $\Delta_{0}(z)^{-1}=$ $\mathcal{L}(d \zeta)(z)$. Para mostrar isto, observe que $\Delta_{0}=I-\mathcal{L}(d \mu)$. Considere agora $\rho$ solução de $\rho=d \mu * \rho-\mu$. Então

$$
\begin{gathered}
\rho-d \mu * \rho=-\mu \\
d \rho-d \mu * d \rho=-d \mu \\
\mathcal{L}(d \rho)(z)-\mathcal{L}(d \mu)(z) \mathcal{L}(d \rho)(z)=-\mathcal{L}(d \mu)(z) \\
{[I-\mathcal{L}(d \mu)(z)] \mathcal{L}(d \rho)(z)=-\mathcal{L}(d \mu)(z)} \\
I+\Delta_{0}(z) \mathcal{L}(d \rho)(z)=\Delta_{0}(z) .
\end{gathered}
$$

de onde segue que

$$
\Delta_{0}(z)^{-1}=I-\mathcal{L}(d \rho)(z)=\mathcal{L}\left(d\left(h_{0}-\rho\right)\right)(z)=\mathcal{L}(d \zeta)(z)
$$

com $\zeta=h_{0}-\rho$. Após estas considerações, podemos escrever

$$
\begin{array}{r}
\Delta_{0}(z)^{-1}\left(f(0)+\int_{0}^{r} e^{-z \theta} d f(\theta)\right)=\mathcal{L}(d \zeta)(z) z \mathcal{L}(f)(z) \\
=z \mathcal{L}(d \zeta * f)(z)=\mathcal{L}(d \zeta * d f)(z)=\mathcal{L}(d \xi)(z)
\end{array}
$$

onde $\xi=d \zeta * f$. Mostremos agora que

$$
\lim _{t \rightarrow \infty} \lim _{N \rightarrow \infty} \int_{-N}^{N} \frac{e^{i \omega t}}{\gamma+i \omega} d \omega=0
$$


Finalmente, usando o Lema 3.14, calculamos o limite da integral dada em (3.30)

$$
\begin{aligned}
\lim _{t \rightarrow \infty} \lim _{N \rightarrow \infty} & \int_{-N}^{N} \frac{e^{i \omega t}}{\gamma+i \omega} \Delta_{0}(\gamma+i \omega)^{-1}\left(f(0)+\int_{0}^{r} e^{-(\gamma+i \omega) \theta} d f(\theta)\right) d \omega \\
& =\lim _{t \rightarrow \infty} \lim _{N \rightarrow \infty} \int_{-N}^{N} \frac{e^{i \omega t}}{\gamma+i \omega}\left(\int_{0}^{r} e^{-(\gamma+i \omega) \theta} d \xi(\theta)\right) d \omega \\
& =\lim _{t \rightarrow \infty} \lim _{N \rightarrow \infty} \int_{0}^{r} e^{-\gamma \theta}\left(\int_{-N}^{N} \frac{e^{i \omega(t-\theta)}}{\gamma+i \omega} d \omega\right) d \xi(\theta) \\
& =\int_{0}^{r} e^{-\gamma \theta}\left(\lim _{t \rightarrow \infty} \lim _{N \rightarrow \infty} \int_{-N}^{N} \frac{e^{i \omega(t-\theta)}}{\gamma+i \omega} d \omega\right) d \xi(\theta)=0 .
\end{aligned}
$$

De (3.31), (3.32), (3.33) e de (3.34), obtemos o resultado.

Lema 3.16. Seja $\lambda$ um zero de $\operatorname{det} \Delta(z)$ de ordem $m$. Então

$$
\operatorname{Res}_{z=\lambda} e^{z t} Q(z)=p(t) e^{\lambda t},
$$

onde $p$ é um polinômio com valores em $\mathbb{C}^{n}$ em $t$ de grau menor ou igual a $m-1$.

Demonstração. Em uma vizinhança de $z=\lambda$, temos as seguintes expansões em séries de Laurent

$$
\begin{gathered}
\Delta(z)^{-1}=\frac{1}{\operatorname{det} \Delta(z)} \operatorname{adj} \Delta(z)=\sum_{k=-m}^{\infty}(z-\lambda)^{k} A_{k}, \\
f(0)+\int_{0}^{r} e^{-z \theta} d f(\theta)=\sum_{k=0}^{\infty}(z-\lambda)^{k} v_{k}, \\
e^{z t}=e^{\lambda t} e^{(z-\lambda) t}=e^{\lambda t} \sum_{k=0}^{\infty} \frac{t^{k}}{k !}(z-\lambda)^{k} .
\end{gathered}
$$

Deste modo, podemos escrever $e^{z t} Q(z)$ como série de Laurent em torno de $z=\lambda$ da forma abaixo

$$
e^{z t} Q(z)=e^{\lambda t}\left(\sum_{k=0}^{\infty} \frac{t^{k}}{k !}(z-\lambda)^{k}\right)\left(\sum_{k=0}^{\infty}(z-\lambda)^{k} v_{k}\right)\left(\sum_{k=-m}^{\infty}(z-\lambda)^{k} A_{k}\right) .
$$

Como o resíduo em $z=\lambda$ é igual ao coeficiente do termo $(z-\lambda)^{-1}$ da série de Laurent de $e^{z t} Q(z)$, segue o resultado. 
Agora estamos prontos para formular o nosso principal resultado.

Teorema 3.17. Seja x uma solução da EDF dada por (2.1)-(2.2), sujeita à condição inicial $x_{0}=\varphi$, onde $\mu$ satisfaz a condição $(J)$. Seja $\gamma>a_{M}$ tal que $\gamma \neq 0$ e para $z$ na reta $\operatorname{Re} z=\gamma \operatorname{det} \Delta(z) \neq 0$ e $\operatorname{det} \Delta_{0}(z) \neq 0$. Então temos a expansão assintótica

$$
x(t)=\sum_{j=1}^{l} p_{j}(t) e^{\lambda_{j} t}+o\left(e^{\gamma t}\right), \quad t \rightarrow \infty
$$

onde $\lambda_{1}, \ldots, \lambda_{l}$ são os zeros de $\operatorname{det} \Delta(z)$ com parte real maior que $\gamma$ e $p_{j}(t)$ são polinômios em $t$ com valores em $\mathbb{C}^{n}$ com grau menor ou igual a $m_{j}-1$, onde $m_{j}$ é a multiplicidade de $\lambda_{j}$ como zero de $\operatorname{det} \Delta_{0}(z)$.

Demonstração. A idéia da prova é dada na discussão anterior ao Lema de Riemann-Lebesgue. Para $\gamma_{1}$ suficientemente grande, obtemos, do Teorema 2.17 que

$$
x(t)=\frac{1}{2 \pi i} \int_{\left(\gamma_{1}\right)} e^{z t} Q(z) d z .
$$

Da equação (3.26), temos que

$$
\frac{1}{2 \pi i} \int_{\left(\gamma_{1}\right)} e^{z t} Q(z) d z=\sum_{j=1}^{m} \operatorname{Res}_{z=\lambda_{j}} e^{z t} Q(z)+\frac{1}{2 \pi i} \int_{(\gamma)} e^{z t} Q(z) d z .
$$

Do Lema 3.15 temos que

$$
\frac{1}{2 \pi i} \int_{(\gamma)} e^{z t} Q(z) d z=\mathrm{o}\left(e^{\gamma t}\right) \quad \text { para } \quad t \rightarrow \infty
$$

e do Lema 3.16, temos a forma polinomial para o resíduo em $z=\lambda_{j}$

$$
\operatorname{Res}_{z=\lambda_{j}} e^{z t} Q(z)=p_{j}(t) e^{\lambda_{j} t}
$$

Então, combinando estes resultados temos

$$
x(t)=\sum_{j=1}^{l} p_{j}(t) e^{\lambda_{j} t}+\mathrm{o}\left(e^{\gamma t}\right) \quad \text { para } \quad t \rightarrow \infty
$$

como queríamos demonstrar. 


\section{Referências Bibliográficas}

[1] Bellman, R., And Cooke, K. L. Differential-difference equations. Academic Press, New York, 1963.

[2] Conway, J. B. Functions of one complex variable, second ed., vol. 11 of Graduate Texts in Mathematics. Springer-Verlag, New York, 1978.

[3] Diekmann, O., van Gils, S. A., Verduyn Lunel, S. M., And Walther, H.-O. Delay equations, vol. 110 of Applied Mathematical Sciences. Springer-Verlag, New York, 1995. Functional, complex, and nonlinear analysis.

[4] Frasson, M. Large time behaviour of neutral delay systems. PhD thesis, Leiden University, 2005.

[5] Hale, J. K., AND Verduyn Lunel, S. M. Introduction to functional-differential equations, vol. 99 of Applied Mathematical Sciences. Springer-Verlag, New York, 1993.

[6] Hewitt, E., And Stromberg, K. Real and abstract analysis. A modern treatment of the theory of functions of a real variable. SpringerVerlag, New York, 1965.

[7] Royden, H. L. Real analysis, third ed. Macmillan Publishing Company, New York, 1988.

[8] Rudin, W. Real and complex analysis, third ed. McGraw-Hill Book Co., New York, 1987. 
[9] Salamon, D. Control and observation of neutral systems, vol. 91 of Research Notes in Mathematics. Pitman (Advanced Publishing Program), Boston, MA, 1984.

[10] Verduyn Lunel, S. M. Exponential type calculus for linear delay equations, vol. 57 of $C W I$ Tract. Stichting Mathematisch Centrum Centrum voor Wiskunde en Informatica, Amsterdam, 1989.

[11] Widder, D. V. The Laplace Transform. Princeton Mathematical Series, v. 6. Princeton University Press, Princeton, N. J., 1941. 